\title{
The MarR-Type Repressor MhqR Confers Quinone and Antimicrobial Resistance in Staphylococcus aureus
}

\author{
Verena Nadin Fritsch,, Vu Van Loi,, Tobias Busche,, ${ }^{1,2}$ Anna Sommer,, Karsten Tedin,, Dennis J. Nürnberg, ${ }^{4}$ \\ Jörn Kalinowski, ${ }^{2}$ Jörg Bernhardt, ${ }^{5}$ Marcus Fulde, ${ }^{3}$ and Haike Antelmann ${ }^{1}$
}

\begin{abstract}
Aims: Quinone compounds are electron carriers and have antimicrobial and toxic properties due to their mode of actions as electrophiles and oxidants. However, the regulatory mechanism of quinone resistance is less well understood in the pathogen Staphylococcus aureus.

Results: Methylhydroquinone (MHQ) caused a thiol-specific oxidative and electrophile stress response in the $S$. aureus transcriptome as revealed by the induction of the PerR, QsrR, CstR, CtsR, and HrcA regulons. The SACOL2531-29 operon was most strongly upregulated by MHQ and was renamed as mhqRED operon based on its homology to the Bacillus subtilis locus. Here, we characterized the MarR-type regulator MhqR (SACOL2531) as quinone-sensing repressor of the mhqRED operon, which confers quinone and antimicrobial resistance in $S$. aureus. The $m h q R E D$ operon responds specifically to MHQ and less pronounced to pyocyanin and ciprofloxacin, but not to reactive oxygen species (ROS), hypochlorous acid, or aldehydes. The MhqR repressor binds specifically to a 9-9 bp inverted repeat (MhqR operator) upstream of the mhqRED operon and is inactivated by MHQ in vitro, which does not involve a thiol-based mechanism. In phenotypic assays, the $m h q R$ deletion mutant was resistant to MHQ and quinone-like antimicrobial compounds, including pyocyanin, ciprofloxacin, norfloxacin, and rifampicin. In addition, the $m h q R$ mutant was sensitive to sublethal ROS and $24 \mathrm{~h}$ post-macrophage infections but acquired an improved survival under lethal ROS stress and after long-term infections.

Innovation: Our results provide a link between quinone and antimicrobial resistance via the MhqR regulon of S. aureus.

Conclusion: The MhqR regulon was identified as a novel resistance mechanism towards quinone-like antimicrobials and contributes to virulence of $S$. aureus under long-term infections. Antioxid. Redox Signal. 31, 1235-1252.
\end{abstract}

Keywords: Staphylococcus aureus, MhqR, QsrR, quinones, antimicrobial resistance

\section{Introduction}

STAPHYLOCOCCUS AUREUS IS a major human patho$\checkmark$ gen, which can cause several diseases including lifethreatening systemic and chronic infections, such as sepsis, necrotizing pneumonia, or endocarditis $(3,8,47)$.The increasing prevalence of multiple antibiotic resistant strains, such as methicillin-resistant $S$. aureus, leads to treatment failure and high mortality rates $(15,54)$. Understanding the defense and resistance mechanisms of $S$. aureus to antibiotics and the host immune response, including reactive oxygen species (ROS) and reactive electrophilic species, will lead to the discovery of novel resistance mechanisms and potential new drug targets to combat multiple antimicrobial resistance.

\footnotetext{
${ }^{1}$ Institute of Biology-Microbiology, Freie Universität Berlin, Berlin, Germany.

${ }^{2}$ Center for Biotechnology, Bielefeld University, Bielefeld, Germany.

${ }^{3}$ Institute of Microbiology and Epizootics, Freie Universität Berlin, Berlin, Germany.

${ }^{4}$ Institute of Experimental Physics, Freie Universität Berlin, Berlin, Germany.

${ }^{5}$ Institute for Microbiology, University of Greifswald, Greifswald, Germany.
}

(C) Verena Nadin Fritsch et al. 2019; Published by Mary Ann Liebert, Inc. This Open Access article is distributed under the terms of the Creative Commons License (http://creativecommons.org/licenses/by/4.0), which permits unrestricted use, distribution, and reproduction in any medium, provided the original work is properly cited. 


\section{Innovation}

The adaptation strategies of Staphylococcus aureus toward reactive oxygen species and reactive electrophilic species are not fully understood, which are required for the successful infection and establishment of antibiotics resistance. In this work, we characterized the novel MhqR repressor as important quinone-sensing and regulatory mechanism in $S$. aureus, which controls quinone detoxification genes and conferred resistance to quinones and quinone-like antimicrobial compounds, including fluoroquinolones (ciprofloxacin, norfloxacin), rifampicin, and pyocyanin. The $m h q R$ mutation further caused an increased survival of $S$. aureus during long-term macrophage infections, and thus, the enzymes of MhqR regulon could be possible drug targets.

Quinones are essential lipid electron carriers of the aerobic and anaerobic respiratory chain in bacteria (e.g., ubiquinone and menaquinone) $(31,39,71)$. However, many natural antimicrobial compounds contain quinone-like structures that are encountered as exogenous sources of quinone stress in pathogenic bacteria, such as the fungal 6-brom-2-vinyl-chroman-4-on (55) or the plant-derived 1,4-naphthoquinone lapachol (32). The toxic effect of quinones is caused by their electrophilic and oxidative modes of actions $(35,52,57)$. Quinones have electrondeficient carbon centers and react as electrophiles with the nucleophilic thiol groups of cysteines via irreversible thiol-Salkylations, leading to aggregation and depletion of thiolcontaining proteins in the proteome (43). As oxidants, quinones can form highly reactive semiquinone radicals that subsequently promote ROS generation, such as superoxide anions, which in turn cause reversible thiol oxidations in proteins $(7,35,52,57)$.

In Bacillus subtilis, two MarR/DUF24 family regulators YodB and CatR as well as the MarR-type repressor MhqR respond to quinones and the azo compound diamide and control together paralogous quinone or azo compound reductases (AzoR1, AzoR2), nitroreductases (YodC, MhqN), and ringcleavage dioxygenases (MhqA, MhqE, MhqO, CatE) for quinone detoxification $(1,2,13,29,42,69)$. The YodB- and MhqRregulated quinone reductases have been shown to confer additive resistance to quinones and diamide in B. subtilis and function in quinone and diamide reduction to hydroquinones and dimethylurea, respectively. The thiol-dependent dioxygenases catalyze the ring cleavage of quinone- $S$-adducts formed by reaction with low-molecular-weight thiols, such as bacillithiol (BSH) (9). Apart from its role in detoxification of exogenous quinones, the catechol 2,3-dioxygenase $\mathrm{CatE}$ was recently shown to function in recycling of the endogenous catecholate siderophore bacillibactin under iron limitation in B. subtilis (65).

Furthermore, the $m h q R$ mutant supported the growth of cell wall-deficient L-forms in $B$. subtilis, which are resistant to $\beta$-lactam antibiotics and promote persister formation (17, 34). The constitutive expression of quinone detoxification genes in the $m h q R$ mutant was suggested to decrease respiratory chain activity and to limit ROS production as mechanism of L-form growth (34).

YodB and CatR are redox-sensing repressors that sense and respond directly to quinones by a redox-switch mechanism involving thiol oxidation at the conserved Cys6 and Cys 7 residues, respectively $(12,13)$. The YodB repressor forms intermolecular disulfides between Cys6 and the Cterminal Cys101 or Cys108 in the opposing subunits of the YodB dimer under quinone and diamide stress in vitro and in vivo $(12,41)$. However, the mechanism of MhqR regulation under quinone stress is unknown thus far and may not involve a thiol-switch mechanism (69).

In S. aureus, the YodB homologue QsrR has been ascribed to be implicated in quinone detoxification, which controls related quinone reductases and a nitroreductase, an flavin mononucleotide-linked monooxygenase, and thiol-dependent dioxygenases (33). The crystal structure of quinone-modified QsrR has been resolved, and the redox-regulatory mechanism was shown to involve thiol- $S$-alkylation of the conserved Cys5 by quinones in vitro (33). Importantly, the QsrR regulon was essential for the pathogenicity of $S$. aureus leading to reduced phagocytosis and increased resistance against killing by bone marrow-derived macrophages (33).

In this work, we aimed to further investigate the quinonestress-specific response in $S$. aureus to elucidate novel mechanisms of redox signaling and antimicrobial resistance. Using RNA-seq transcriptomics, we identified the $m h q R E D$ operon as most strongly induced by methylhydroquinone (MHQ) in S. aureus, which is controlled by SACOL 2531 (MhqR), a close homolog to MhqR of B. subtilis (69). Our results demonstrate that the $m h q R E D$ operon confers resistance to quinones and quinone-like antimicrobials, including pyocyanin, ciprofloxacin, norfloxacin, and rifampicin. Due to the increasing prevalence of multiple antibiotic resistant $S$. aureus isolates, these results are important to understand the underlying mechanisms of antimicrobial resistance.

\section{Results}

MHQ elicits a thiol-specific oxidative, electrophile, and metal stress response in the RNA-seq transcriptome of $\mathrm{S}$. aureus

To investigate the quinone-stress-specific response of $S$. aureus COL, we analyzed the changes in the RNA-seq transcriptome after exposure to sublethal MHQ stress $(45 \mu M)$ (Supplementary Fig. S1) (30, 44). For significant fold-changes, the $M$-value cutoff ( $\log 2$-fold-change MHQ $v s$. control) of \pm 0.6 was chosen (adjusted $p$-value $\leq 0.05$ ). In total, 730 transcripts were significantly $>1.5$-fold upregulated and 675 were $>1.5$ fold downregulated in the transcriptome of $S$. aureus under MHQ stress (Supplementary Tables S1 and S2). A subset of the most strongly upregulated regulons is displayed in the Voronoi transcriptome treemap (Fig. 1). About 70 genes displayed the highest fold-changes under MHQ stress ranging from 10 to 536 ( $M$-values of 3.3-9), which could be mainly allocated to the TetR, QsrR, PerR, Fur, CtsR, CstR, CsoR, SigB, and GraRS regulons (Figs. 1 and 2 and Supplementary Fig. S2; Supplementary Tables S1 and S2). This indicates that MHQ leads generally to a strong thiol-specific oxidative (PerR), electrophile (QsrR), metal (Fur, CsoR), and cell wall stress response (GraRS, SigB) in $S$. aureus.

Among the top hits was the SACOL2588-89 operon of hypothetical functions (510- to 536-fold) and the QsrR regulon, including SACOL2533 (catE2), SACOL0408-09-10 (catESACOL0409-azoRl), SACOL2534 (frp), and SACOL2020 (yodC) (25- to 121-fold induced). Interestingly, our transcriptome data revealed also a strong (35- to 67-fold) upregulation of the SACOL2531-30-29 operon that encodes 


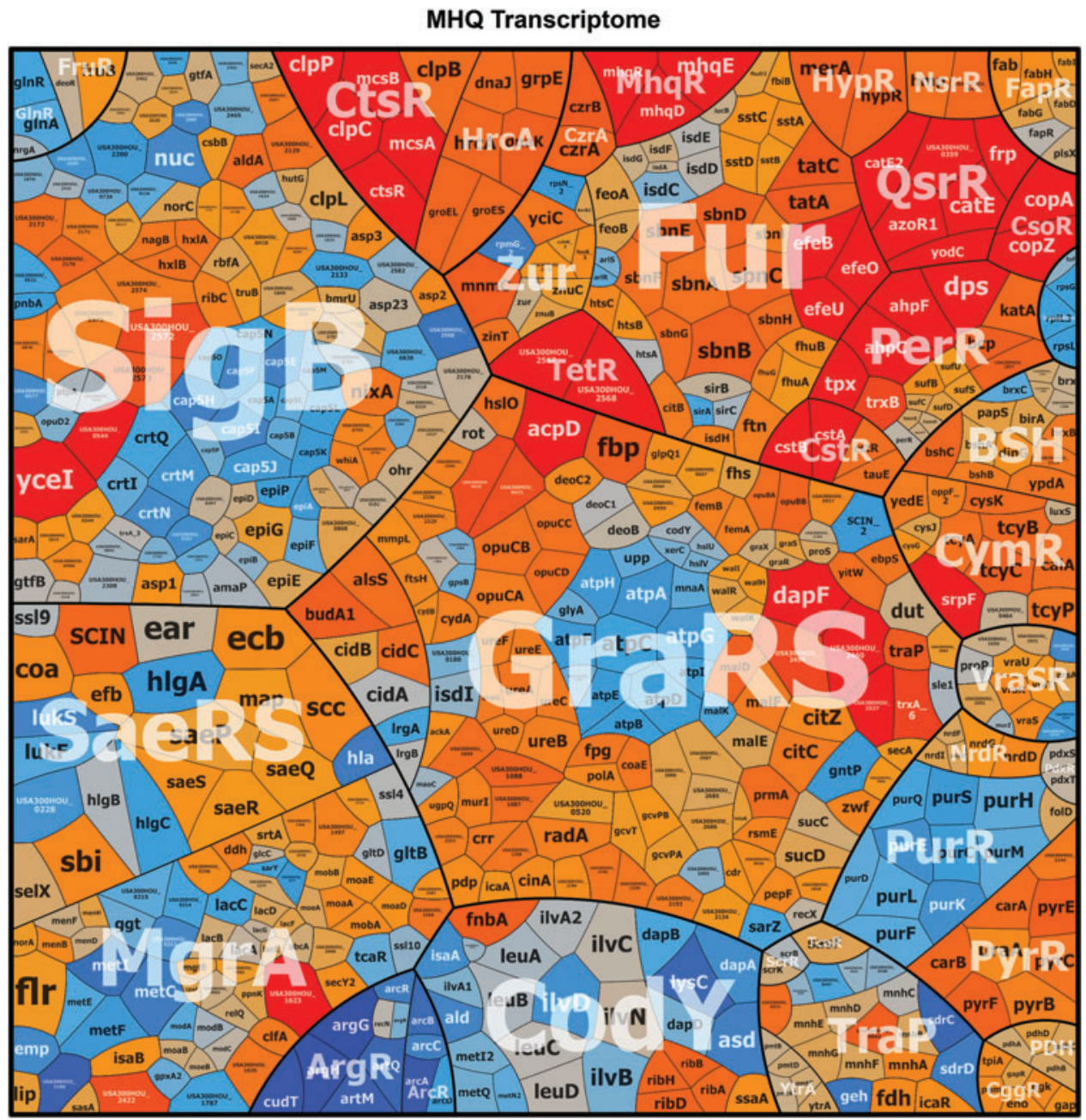

log fold change -4

4

FIG. 1. The transcriptome treemap of Staphylococcus aureus COL under MHQ stress indicates a strong upregulation of the MhqR and QsrR regulons. The transcriptome treemap shows the differential gene expression of $S$. aureus after exposure to $45 \mu M \mathrm{MHQ}$ as $\log 2$-fold-changes ( $M$-values). The genes are classified into operons and regulons based on the RegPrecise database and previous publications $(44,49,72)$. Differential gene expression is visualized using a red-blue color code where red indicates $\log 2$-fold induction and blue indicates repression of transcription under MHQ stress. The quinonestress-specific regulons MhqR and QsrR are most strongly upregulated under MHQ stress in S. aureus COL. The induction of the PerR, CsoR, Fur, HrcA, CtsR, and GraRS regulons reveals an oxidative, electrophile, metal, and cell wall stress response and protein damage in S. aureus. The RNA-seq expression data of the selected highly transcribed genes after MHQ stress and their regulon classifications are listed in Supplementary Table S2. MHQ, methylhydroquinone. Color images are available online.

for the phospholipase/carboxylesterase SACOL2529 (MhqD), the dioxygenase SACOL2530 (MhqE), and the unknown MarR-type regulator SACOL2531. SACOL2531 showed striking homology (39.4\% sequence identity) to the quinonespecific MhqR repressor of B. subtilis (69) and was renamed MhqR in S. aureus (Supplementary Fig. S3A). Thus, the transcriptome results identified QsrR and MhqR as most responsive to MHQ in $S$. aureus, which resembles the quinone stress response in B. subtilis (1, 18, 29, 42, 55, 68, 69).

We have previously analyzed the transcriptome signature of S. aureus USA300 in response to the strong oxidant sodium hypochlorite $(\mathrm{NaOCl})$ and the antimicrobial surface coating $\mathrm{AgXX}^{\circledR}$, which causes ROS formation, such as hydroxyl radicals $(44,72)$. Our RNA-seq data after MHQ treatment showed a similar expression profile by the strong induction of the PerR, QsrR, Fur, CsoR, HrcA, CtsR, and GraRS regulons, as observed under $\mathrm{NaOCl}$ and AGXX stress $(44,72)$. This signature is indicative for a thiol-specific oxidative, electrophile, metal, and cell wall stress response as well as protein damage.

Specifically, MHQ leads to induction of the CtsR-controlled Clp proteases, including $\operatorname{clpP}$ (17-fold) and the $\operatorname{cts} R$-mcsA$m c s B$-clp $C$ operon (16- to 21-fold) involved in protein quality control and proteolytic degradation of quinone-aggregated proteins (1). The PerR, Fur, and CsoR regulons function in ROS detoxification, iron or copper homeostasis, and these metalloregulatory proteins have oxidation-sensitive metal binding sites $(5,23)$. The CstR regulon responds to reactive sulfur species and thiol persulfides (48). Transcription of the genes for cysteine and bacillithiol metabolism ( $c y s K, b s h A$ operon, $b s h B, b s h C, b r x B$, and $y p d A$ ) was 1.6- to 5.6-fold elevated by MHQ in $S$. aureus supporting the thiol-reactive mode of action of quinones, which affects the cellular thiol-redox homeostasis (67). About 87 genes 
of the GraRS regulon and parts of the SigB regulon were upregulated by MHQ, which function in the cell wall and general stress response as well as in the oxidative stress defense (21).

However, the SigB-dependent $\operatorname{crtNMQIO}$ operon for staphyloxanthin biosynthesis and the capsule biosynthesis $c a-$ p5ABCDEFGHIJKLMNOP operon were strongly repressed by MHQ (Fig. 1 and Supplementary Tables S1 and S2). Among the downregulated regulons were further the arginine biosynthesis ArgR regulon, including the $\arg B J C D, \operatorname{argHG}$, and $\operatorname{art} Q M$ operons, as well as the arginine catabolic ArcR regulon, controlling the arginine deiminase $\operatorname{arc} C D B A$ operon. In addition, the purine biosynthesis PurR regulon was downregulated by MHQ, which might be attributed to the reduced growth rate under sublethal MHQ (Supplementary Fig. S1). Altogether, the transcriptome signature of MHQ resembles the thiol-specific oxidative, electrophile, and metal stress response and identified the $m h q R E D$ operon as novel quinone-regulatory system that was selected for further study.

\section{The MhqR repressor senses quinones and controls} the specific expression of the mhqRED operon in $\mathrm{S}$. aureus

We conducted RNA-Seq transcriptomics of a $m h q R$ deletion mutant to identify the genes of the MhqR regulon.
The $m h q E$ and $m h q D$ genes were most strongly upregulated (206.5- to 891.4-fold) under control conditions in the $m h q R$ mutant transcriptome, indicating that MhqR represses transcription of the $m h q R E D$ operon in the wild type (Figs. 2 and 3 and Supplementary Fig. S2; Supplementary Table S2). MhqE and MhqD showed $35.4 \%$ and $38.8 \%$ sequence identity to the homologous dioxygenase MhqE and phospholipase/carboxylesterase MhqD of B. subtilis, respectively (Supplementary Fig. S3B). In contrast to $B$. subtilis, MhqR only controls the mhqRED operon in $S$. aureus (Fig. 2 and Supplementary Fig. S2; Supplementary Table S2) (69).

The transcriptome results of the mhqR mutant further revealed that most thiol-specific oxidative and electrophile stress regulons (e.g., HypR, QsrR, and PerR) are expressed at a lower basal level under control conditions in the $m h q R$ mutant compared with the wild type. For example, peroxide scavenging peroxiredoxins and catalases (ahpCF and katA) showed twofold lower basal level expression in the $m h q R$ mutant compared with the wild type (Fig. 2 and Supplementary Fig. S2; Supplementary Table S2). This lower basal expression of antioxidant and quinone detoxification regulons might be due to the quinone-resistant phenotype of the $m h q R$ mutant enabling faster quinone detoxification, which leads to lower basal levels of ROS. Consequently, the
A

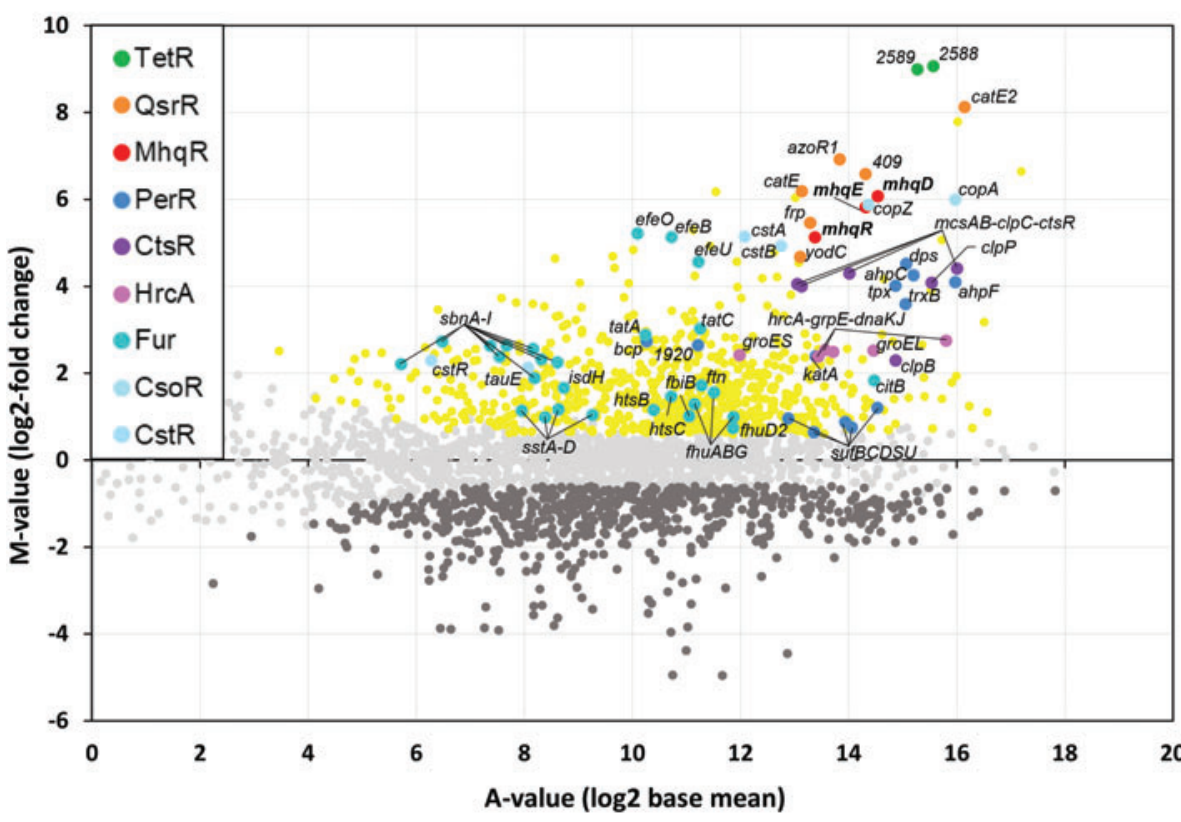

B

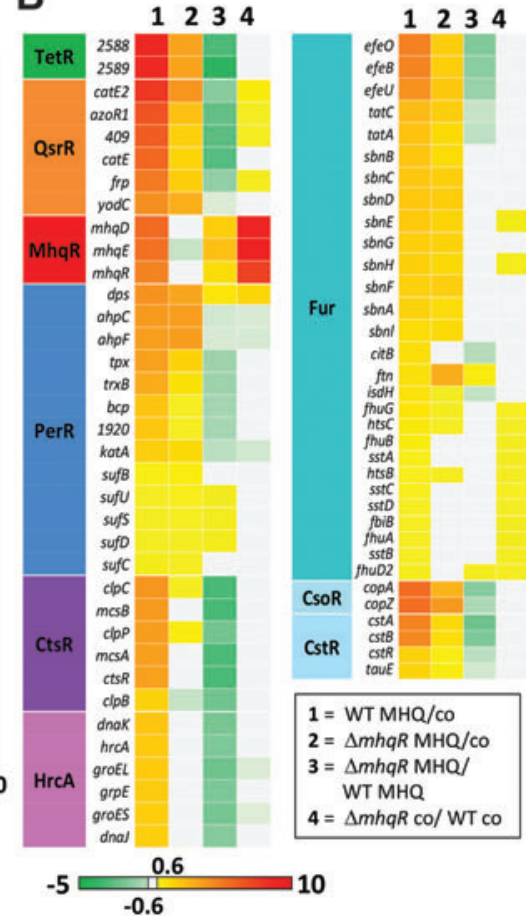

FIG. 2. RNA-seq transcriptomics of $S$. aureus COL wild type and the $m h q R$ mutant under MHQ stress. For RNAseq transcriptomics, $S$. aureus COL and the $m h q R$ mutant were grown in RPMI1640 medium and treated with $45 \mu M$ MHQ stress for $30 \mathrm{~min}$. (A) The gene expression profile of the wild type under MHQ stress is shown as ratio/intensity scatterplot (M/ A-plot), which is based on the differential gene expression analysis using DeSeq2 (46). Colored symbols indicate significantly induced (red, orange, yellow, blue, cyan, violet, green) or repressed (dark gray) transcripts ( $M$-value $\geq 0.6$ or $\leq-0.6 ; p \leq 0.05$ ). Light gray symbols denote transcripts with no fold-changes after MHQ stress $(p>0.05)$. The TetR, QsrR, MhqR, PerR, CtsR, HrcA, Fur, CsoR, and CstR regulons are most strongly upregulated under MHQ stress. (B) The color-coded heat map displays $\log 2$-fold-changes of gene expression between the wild type and the $m h q R$ mutant under control and MHQ. Red and green indicate significantly induced and repressed transcripts $(M$-value $\geq 0.6$ or $\leq-0.6 ; p \leq 0.05)$ in three biological replicates, respectively. The RNA-seq expression data of all genes under MHQ stress and their regulon classifications are listed in Supplementary Tables S1 and S2. Color images are available online. 


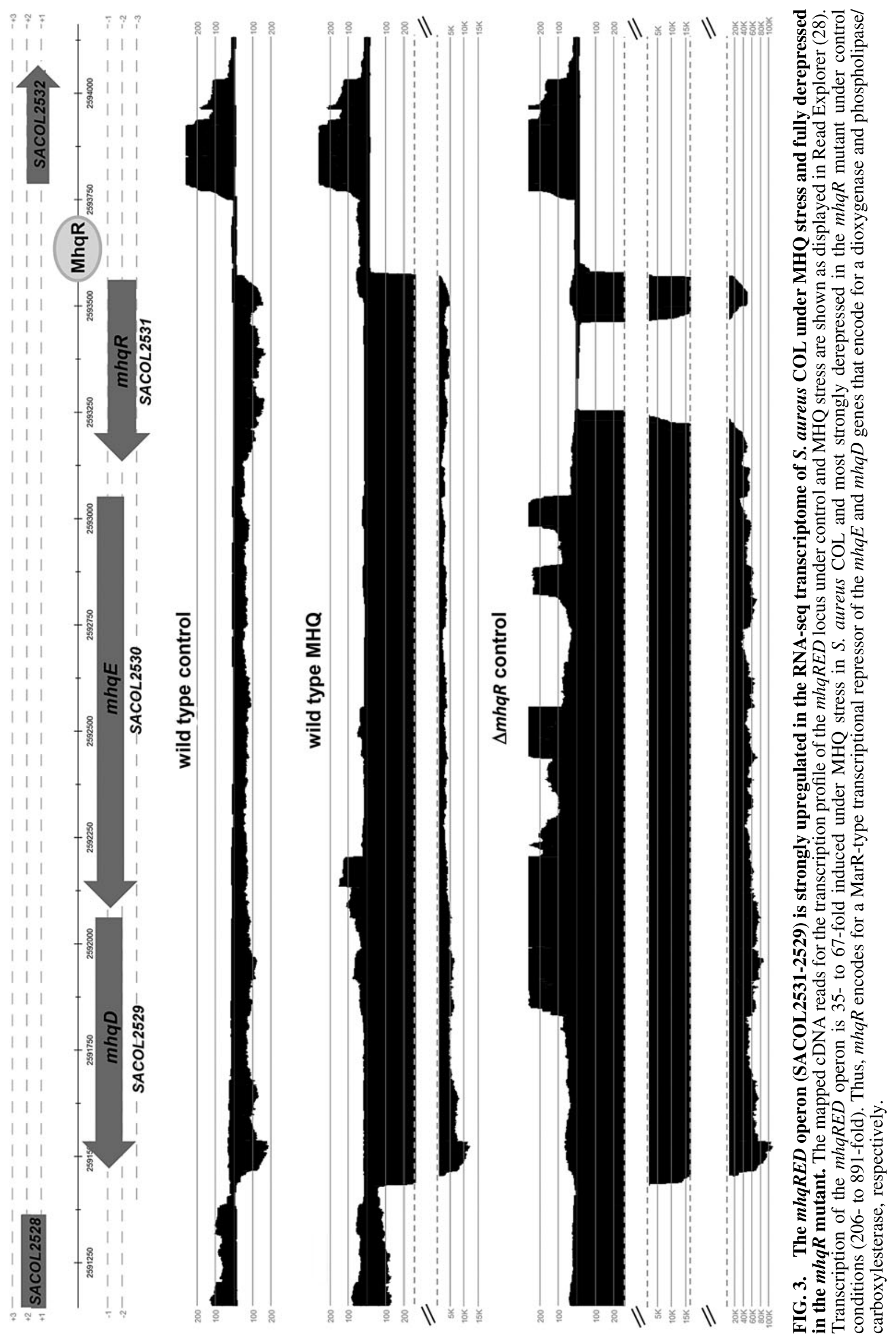


quinone and oxidative stress responsive HypR, QsrR, and PerR regulons and genes required for low molecular weight thiol biosynthesis (Cys, BSH) were only weakly upregulated in the $m h q R$ mutant under MHQ treatment due to its higher tolerance for quinones. Similarly, the $m h q R$ mutant displayed decreased fold-changes under MHQ for the majority of members of the cell wall, sulfide, and metal stress-sensing SigB, GraRS, CsoR, and CstR regulons (Fig. 2 and Supplementary Fig. S2; Supplementary Table S2). Moreover, the expression of the CtsR- and HrcA-controlled protein quality control machinery was $>5$-fold decreased under MHQ stress in the $m h q R$ mutant. In conclusion, constitutive derepression of the MhqR regulon in the $m h q R$ mutant leads to higher quinone detoxification capability, which limits ROS generation and the resulting protein oxidation and damage.

The mhqRED operon responds specifically to quinones and the antimicrobials ciprofloxacin, pyocyanin, and lapachol in S. aureus

Next, we conducted Northern blot analysis to study mhqRED transcription in $S$. aureus COL under different stress conditions and antibiotic treatment, including $45 \mu M \mathrm{MHQ}, 1 \mathrm{~m} M$ $\mathrm{NaOCl}, 2 \mathrm{~m} M$ diamide, $0.75 \mathrm{~m} M$ formaldehyde, $0.5 \mathrm{~m} M$ methylglyoxal, $300 \mu M$ lapachol, $76 \mu M$ pyocyanin, and $90.5 \mu M$ ciprofloxacin (Fig. 4A). The Northern blot results
A
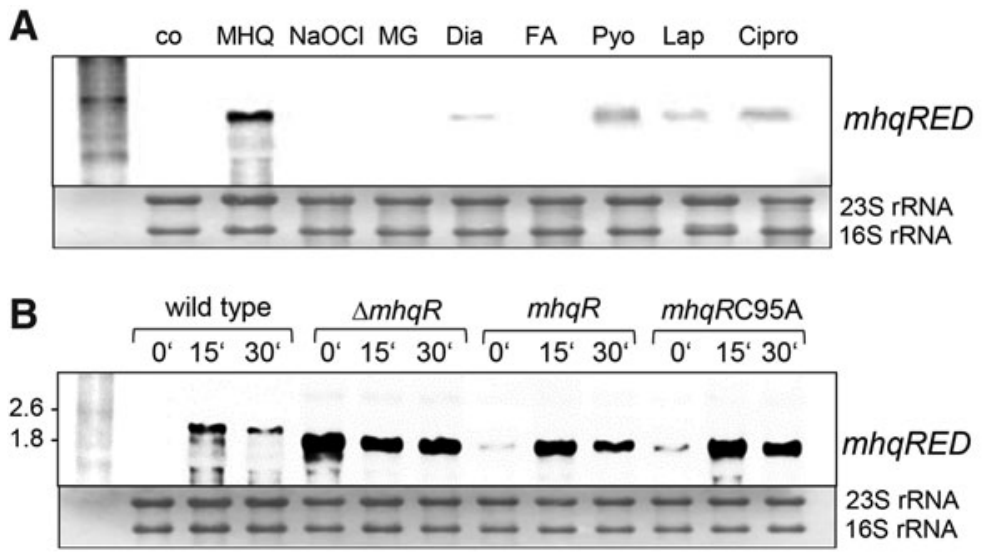

D

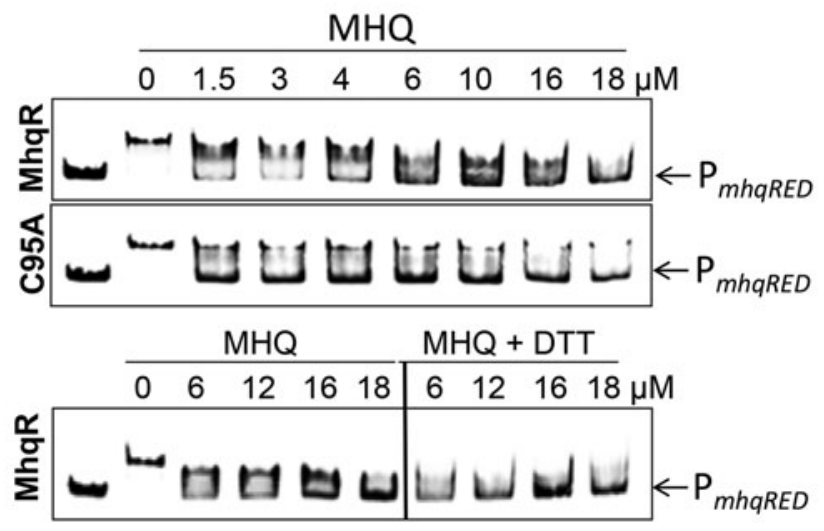

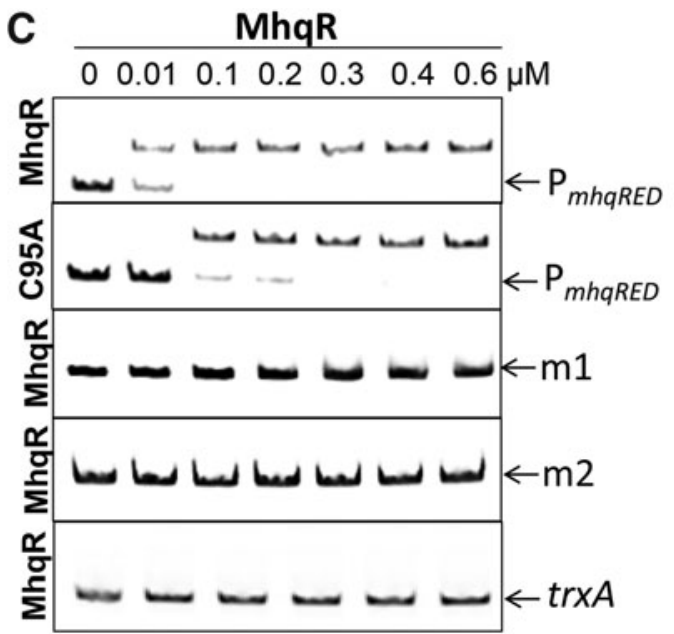

TATCTCGAA ATCGAAATA MhqR operator TATCGCTAA ATCGAAATA m1 TATCTCGAA ATAGG $\underline{\text { AATA }} \mathrm{m} 2$

FIG. 4. Transcriptional induction of the MhqR regulon under quinones, aldehydes, and antimicrobials and the quinone response of MhqR in DNA binding assays in vitro. (A) Transcription of the $m h q R E D$ operon was analyzed using the Northern blots in $S$. aureus COL wild type $30 \mathrm{~min}$ after exposure to $45 \mu M \mathrm{MHQ}, 1 \mathrm{~m} M \mathrm{NaOCl}, 0.5 \mathrm{~m} M$ methylglyoxal (MG), $2 \mathrm{~m} M$ diamide (Dia), $0.75 \mathrm{~m} M$ formaldehyde (FA), $300 \mu M$ lapachol (Lap), $90.5 \mu M$ ciprofloxacin (Cipro), and $76 \mu M$ pyocyanin (Pyo). The compounds were added at an $\mathrm{OD}_{500}$ of 0.5 . The $m h q R E D$ operon responds most strongly to MHQ and less strongly to lapachol, pyocyanin, and ciprofloxacin. (B) The Northern blot analysis was performed with RNA of the wild type, the $m h q R$ mutant, and the $m h q R$ and $m h q R C 95 A$ complemented strains before $(0 \mathrm{~min})$ and 15 and $30 \mathrm{~min}$ after MHQ stress. Cys95 is not required for DNA binding and quinone sensing of MhqR in vivo. The methylene blue stain is the RNA loading control indicating the $16 \mathrm{~S}$ and $23 \mathrm{~S}$ rRNAs. (C) MhqR binds specifically to the mhqRED promoter in vitro. EMSAs were used to analyze the DNA binding activity of increasing amounts $(0.01-0.6 \mu M)$ of MhqR and MhqRC95A proteins to the mhqRED promoter $\left(\mathrm{P}_{\text {mhqRED }}\right)$ in vitro. To test the specificity of binding, two base substitutions were introduced in each half of the inverted repeat, denoted in gray and underlined $(\mathrm{m} 1$ and $\mathrm{m} 2)$. As nonspecific control DNA probe we used the trxA gene. The arrows denote the free DNA probe and the shifted band indicates the DNA-MhqR promoter complex. (D) EMSAs of MhqR and MhqRC95A proteins $(0.6 \mu M)$ to the $m h q R E D$ promoter were performed to study the inactivation of MhqR by increasing amounts of MHQ (1.5-18 $\mu M$ ) leading to the loss of DNA binding. The arrows denote the free mhqRED promoter probe and the shifted band indicates the DNA-MhqR promoter complex. (E) MhqR inactivation by quinones could not be reversed with $1 \mathrm{~m} M$ DTT, which was added to the MhqR-DNA binding reaction 30 min after MHQ addition. Cys95 is not important for MHQ sensing or DNA binding of MhqR in vitro. DTT, dithiothreitol; EMSA, electrophoretic mobility shift assay; $\mathrm{NaOCl}$, sodium hypochlorite. 
revealed that the $m h q R E D$ operon is most strongly induced by MHQ stress but does not respond to $\mathrm{NaOCl}$ and aldehydes. Interestingly, increased transcription of mhqRED operon was also found by the quinone-like antimicrobials, such as ciprofloxacin, pyocyanin, and the 1,4-naphthoquinone lapachol (Fig. 4A; Supplementary Fig. S4). Thus, the MhqR regulon responds specifically to quinones and diverse quinone-like antimicrobials in $S$. aureus, suggesting a function in antimicrobial resistance.

The DNA binding activity of MhqR is inhibited by quinones in vivo and in vitro, which does not involve a thiol-based mechanism

S. aureus MhqR harbors a nonconserved Cys at position 95. To examine the role of Cys95 for DNA binding and quinone sensing, we complemented the $m h q R$ mutant with plasmid-encoded $m h q R$ and the $m h q R C 95 A$ mutant allele. The Northern blot analyses confirmed the constitutive expression of the $1.7 \mathrm{~kb}$ truncated $m h q R E D$-specific mRNA in the $m h q R$ mutant. Complementation of the $m h q R$ mutant with $m h q R$ restored repression of transcription of the mhqRED operon under control conditions and the strong quinone response to wild-type level (Fig. 4B). However, the mhqRC95A mutant also showed the same low basal level transcription and strong responsiveness to MHQ of the $m h q R E D$ operon compared with the wild type and $m h q R$ complemented strain. Thus, the Northern blot data revealed that Cys95 is neither required for DNA binding nor for quinone sensing in vivo.

Electrophoretic mobility shift assays (EMSAs) were used to investigate the DNA binding activity of purified MhqR protein to the mhqRED promoter in vitro. The mhqREDspecific promoter probe covered the region from +32 to -192 relative to the transcription start site (TSS). The gel shift results showed that purified MhqR binds to the mhqRED promoter probe, which is indicated by the band shift in the DNA binding reactions with MhqR (Fig. 4C).

Inspection of the $m h q R E D$ promoter region identified a 9$9 \mathrm{bp}$ imperfect inverted repeat with the sequence TATCTCGAA-aTCGAaATA in position -6 to +12 relative to the TSS +1 (Fig. 5). The inverted repeat overlapping with the TSS was termed as MhqR operator based on its conservation with the MhqR operator upstream of azoR2, mhqNOP, $m h q E D$, and $m h q A$ in B. subtilis (69). To analyze the specific binding of MhqR to the MhqR operator, we exchanged two nucleotides in each half of the inverted repeat $(\mathrm{m} 1: \mathrm{T}$ to $\mathrm{G}$ and $\mathrm{G}$ to $\mathrm{T} ; \mathrm{m} 2$ : $\mathrm{C}$ to $\mathrm{A}$ and $\mathrm{A}$ to $\mathrm{G}$ ) and analyzed the DNA binding activity of MhqR to these mutated promoter probes (Fig. 4C). MhqR was unable to bind to the mutated inverted repeats $\mathrm{m} 1$ and $\mathrm{m} 2$ in vitro. In addition, no band shift was observed in the reaction of MhqR with the nonspecific $\operatorname{trxA}$ DNA probe, further supporting the specific binding of MhqR to the identified operator sequence (Fig. 4C).

Next, we investigated DNA binding and quinone-sensing of MhqR and MhqRC95A proteins. The MhqRC95A protein was able to bind with slightly decreased affinity to the mhqRED promoter probe compared with MhqR (Fig. 4C). Based on the EMSA results, the dissociation constants $\left(K_{\mathrm{d}}\right)$ were calculated as 7.38 and $14.25 \mathrm{n} M$ for $\mathrm{MhqR}$ and MhqRC95A mutant proteins, respectively. Treatment with increasing concentrations of MHQ resulted in complete dissociation of the MhqR and MhqRC95A proteins from the $m h q R E D$ promoter probe with $16-18 \mu M \mathrm{MHQ}$, respectively (Fig. 4D). The addition of dithiothreitol (DTT) to the reaction of quinone-treated MhqR did not restore the DNA binding ability of MhqR, supporting that MhqR inactivation by quinones is not caused by a reversible thiol-switch (Fig. 4E). Thus, the nonconserved Cys95 of MhqR is not required for DNA binding and redox sensing of quinones in vitro, confirming our in vivo Northern blot results. This indicates that inactivation of the MhqR repressor by quinones does not involve a thiol-based mechanism. We speculate that MHQ binds to a specific ligand binding pocket in MhqR as revealed for other ligand binding MarR-type regulators (24), leading to its inactivation and derepression of the $m h q R E D$ operon.

Since no crystal structure of MhqR is available, the structure of $S$. aureus MhqR was modeled based on the template of the crystal structure of the MarR-family regulator ST1710 from Sulfolobus tokodaii (3GFI) using SWISS MODEL $(6,38)$ (Supplementary Fig. S5). MhqR of S. aureus shares $18.2 \%$ sequence identity with ST1710. The crystal structure of the ST1710 dimer was resolved in complex with its promoter DNA and with its ligand sodium salicylate, which is a common inhibitor of MarR proteins (38) (Supplementary Fig. S5A).

Similar to other MarR-type transcription factors, each subunit of the MhqR dimer is composed of six $\alpha$-helices and two $\beta$-sheets, arranged as $\alpha 1-\alpha 2-\alpha 3-\alpha 4-\beta 1-\beta 2-\alpha 5-\alpha 6$ (Supplementary Fig. S5B). The $\alpha 1, \alpha 5$, and $\alpha 6$ helices form the dimer interface of the two MhqR subunits, and the DNA binding domain is composed of the $\alpha 2, \alpha 3, \alpha 4$ helices and the $\beta 1, \beta 2$ wing, known as winged helix-turn-helix (wHTH) DNA binding motif $(16,24)$. In the ST1710 structure complexed with salicylate, the ligand was coordinated by Y37 and Y111 of one subunit and A16, K17, and R20 of the opposing subunit of the dimer. This ligand binding pocket is located at the interface between the dimerization domains and the wHTH motif as described for other MarR-type regulators $(24,38)$. However, none of the salicylate coordinating tyrosine, lysine, or arginine residues of ST1710 is conserved in MhqR (Supplementary Fig. S5B). Thus, the mechanism of quinone binding in MhqR and the resulting conformational changes remain to be elucidated.

\section{The MhqR regulon confers resistance to $M H Q$ and quinone-like antimicrobials in $\mathrm{S}$. aureus}

Next, we were interested whether the MhqR regulon is involved in quinone and antimicrobial resistance mechanisms. The growth and survival phenotypes of the $m h q R$ mutant were analyzed under MHQ stress and after treatment with different antimicrobial compounds, including pyocyanin, ciprofloxacin, norfloxacin, rifampicin, and lapachol (Figs. 6 and 7). The $m h q R$ mutant showed high resistance to $50 \mu M$ MHQ and was not inhibited in growth compared with the wild type and $m h q R$ complemented strain (Fig. 6A and Supplementary Fig. S6A). In addition, the $m h q R$ mutant displayed two- to threefold increased survival in killing assays with lethal doses of 100-250 $\mu M$ MHQ (Fig. 6C).

Treatment of the $m h q R$ mutant with the antimicrobials pyocyanin, ciprofloxacin, norfloxacin, and rifampicin resulted in slightly improved growth at sublethal doses and significantly enhanced survival in killing assays with lethal concentrations of the antimicrobial compounds (Figs. 6D-I 


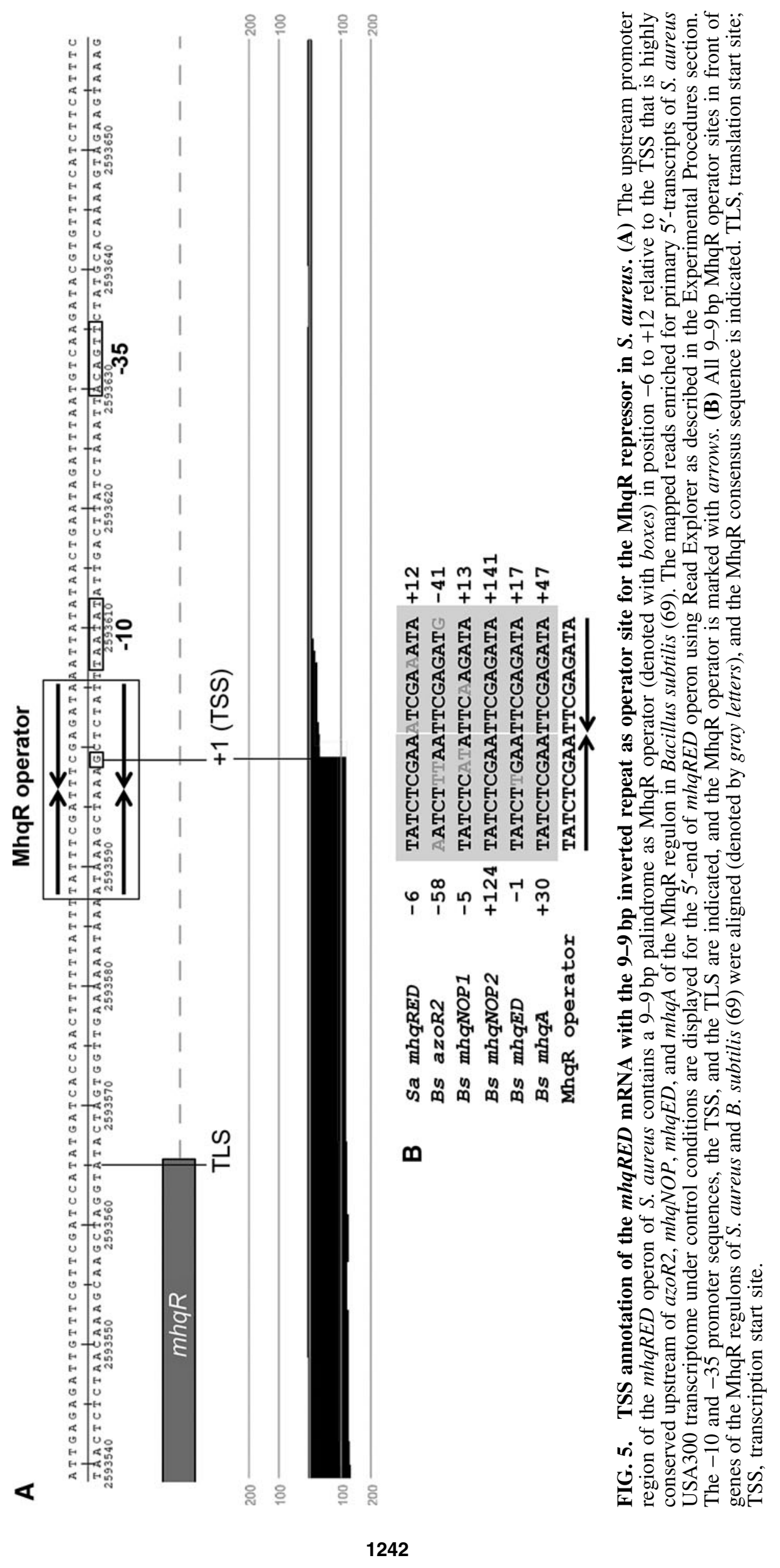



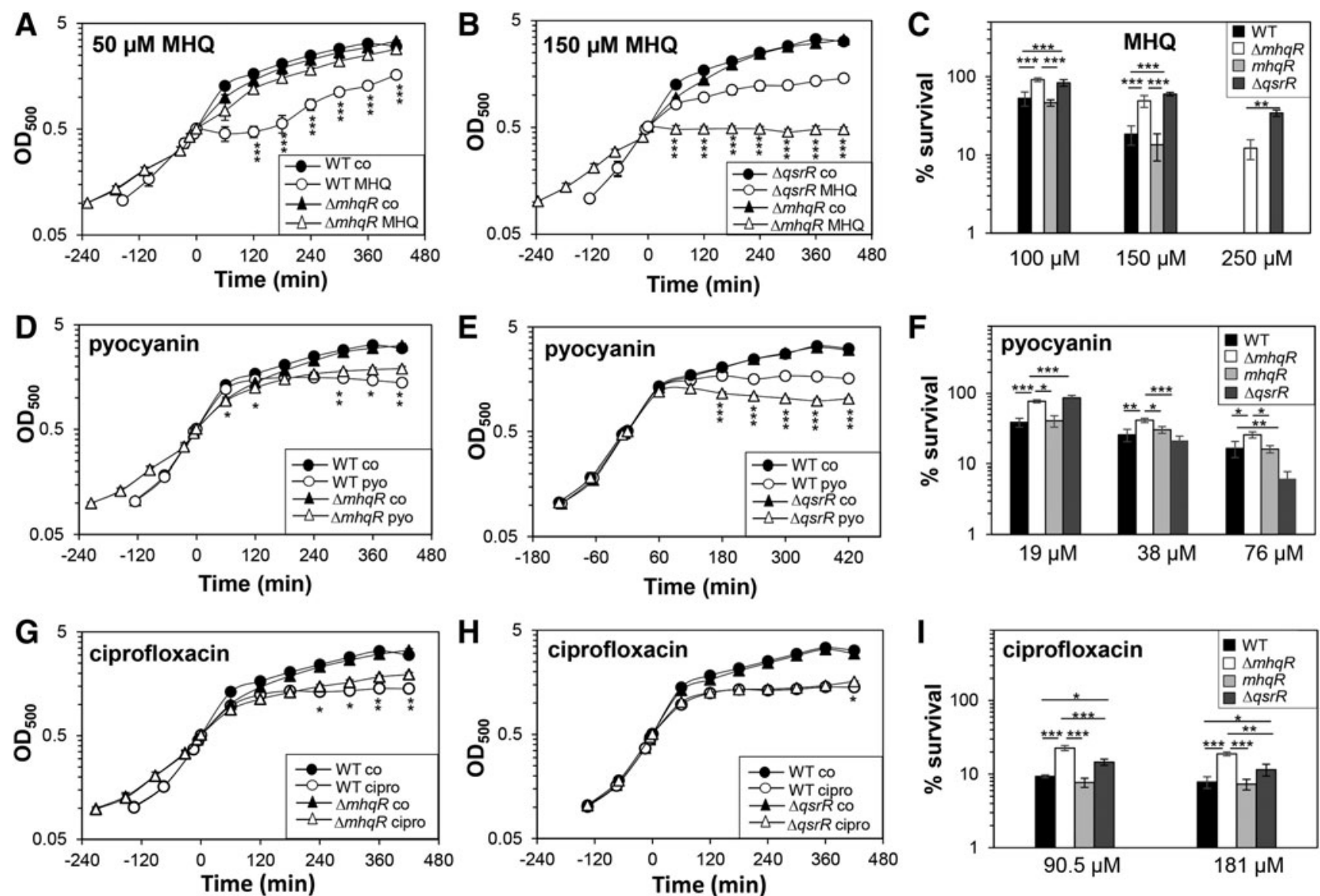

FIG. 6. The MhqR and QsrR regulons confer resistance to MHQ and the antimicrobials pyocyanin and ciprofloxacin. (A, B, D, E, G, and $\mathbf{H}$ ) For the growth curves, $S$. aureus COL wild type, $m h q R$ and $q s r R$ mutants, as well as the $m h q R$ complemented strain $(m h q R)$ were grown in RPMI until an $\mathrm{OD}_{500}$ of 0.5 and treated with 50 and $150 \mu M$ MHQ, $76 \mu M$ pyocyanin, and $90.5 \mu M$ ciprofloxacin. (C, F, and I) Survival assays were performed by treatment with sublethal and lethal doses and plating $100 \mu \mathrm{L}$ of serial dilutions onto LB agar plates after $4 \mathrm{~h}$ of stress exposure. The survival rates of CFUs for the treated samples were calculated relative to the control, which was set to $100 \%$. The $m h q R$ and $q s r R$ mutants are significantly more resistant to MHQ, pyocyanin, and ciprofloxacin, which could be restored to wild-type levels in the $m h q R$ complemented strain. The results are from four biological replicates. Error bars represent the standard deviation. $* p<0.05 ; * * p<0.01 ; * * * p<0.001$. CFU, colony-forming unit; LB, Luria-Bertani.

and 7A-D). These antibiotic resistant phenotypes of the $m h q R$ mutant could be restored back to wild-type level in the $m h q R$ complemented strain (Supplementary Fig. S6B-E). However, the $m h q R$ mutant was significantly impaired in growth and survival after treatment with the 1,4-naphthoquinone lapachol (Fig. 7E, F). These results indicate that the MhqR regulon protects $S$. aureus against benzoquinones, and many other antimicrobials that contain quinone-like structures, but not against naphthoquinones.

The MhqR and QsrR regulons contribute independently to quinone and antimicrobial resistance

Apart from MhqR, the MarR/DUF24-type regulator QsrR was shown to mediate resistance to quinones and pyocyanin in $S$. aureus $(33,56)$. Thus, we compared the growth and survival phenotypes of the $m h q R$ and $q s r R$ mutants in response to MHQ, ciprofloxacin, norfloxacin, rifampicin, and pyocyanin (Figs. 6 and 7). The MhqR and QsrR regulons conferred significant resistance to MHQ, rifampicin, and the fluoroquinolone ciprofloxacin, but not to the same extent. The $q s r R$ mutant was able to grow even with lethal doses of $150 \mu \mathrm{M} \mathrm{MHQ}$, which resulted in growth inhibition of the $m h q R$ mutant (Fig. 6B). In survival assays, both mutants exhibited the same level of approximately two- to threefold increased resistance toward MHQ relative to the parent (Fig. 6C). Thus, the QsrR regulon conferred higher resistance to quinones than the $m h q R$ mutant.

In contrast, the $m h q R$ mutant showed higher ciprofloxacin resistance in growth assays and improved survival under ciprofloxacin, norfloxacin, and rifampicin treatment compared with the qsrR mutant (Figs. 6G-I and 7A-D).The MhqR and QsrR regulons contributed to a significant protection under low doses of $19 \mu M$ pyocyanin (Fig. 6D-F). However, only the MhqR regulon protected against high pyocyanin concentrations (38-76 $\mu M)$ in killing assays. In contrast, the $q s r R$ mutant was significantly more susceptible than the wild type at higher pyocyanin doses (Fig. 6E, F). These results point to independent roles of $\mathrm{MhqR}$ and QsrR as players in the quinone stress response. While the $\mathrm{QsrR}$ regulon 

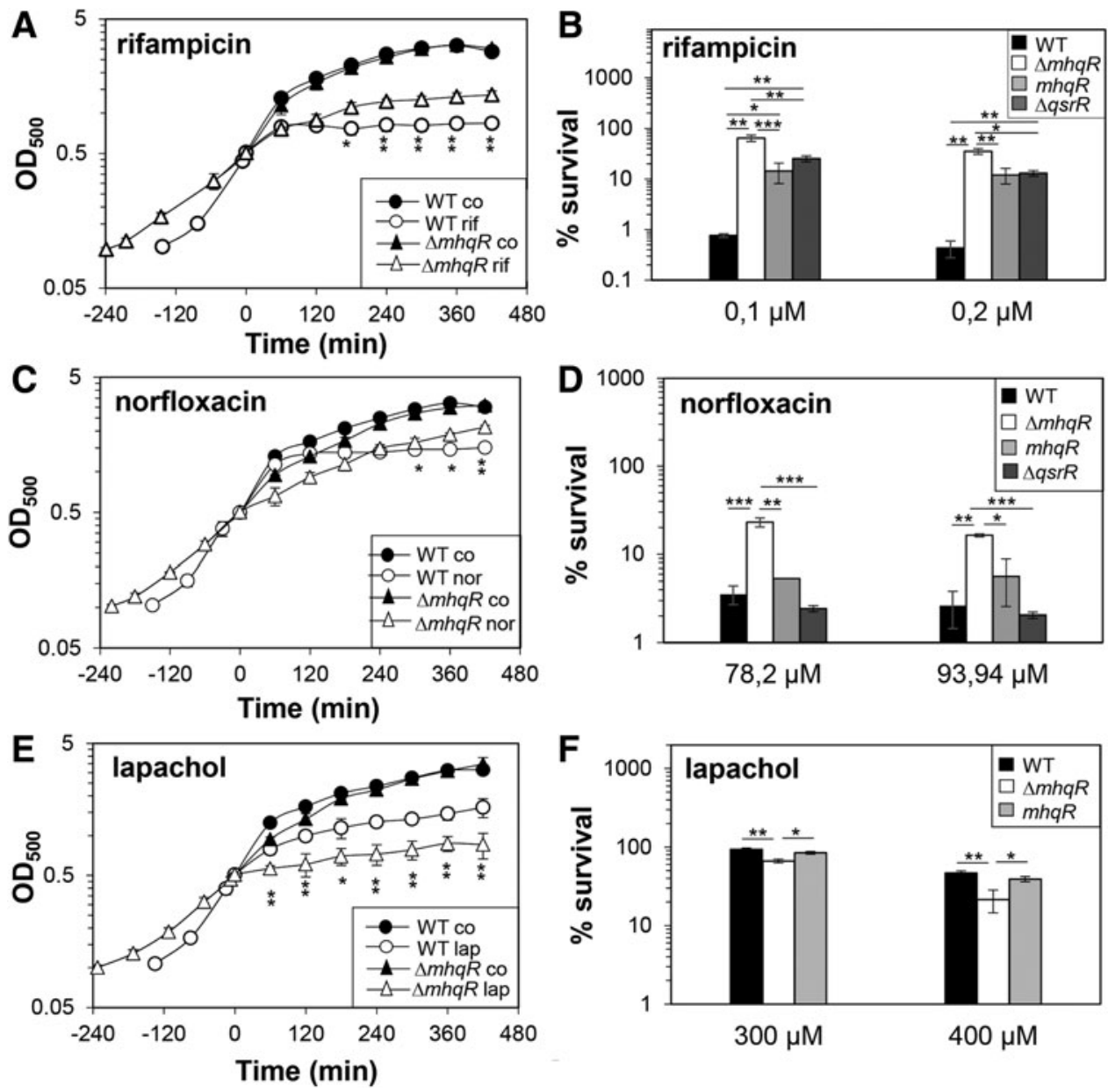

FIG. 7. The $m h q R$ mutant is resistant to rifampicin and norfloxacin but impaired in survival after lapachol stress. $(\mathbf{A}, \mathbf{C}, \mathbf{E})$ For the growth curves, $S$. aureus COL wild type, the $m h q R$ and $q s r R$ mutants, and the $m h q R$ complemented strain $(m h q R)$ were grown in RPMI until an $\mathrm{OD}_{500}$ of 0.5 and treated with $0.05 \mu M$ rifampicin, $62.6 \mu M$ norfloxacin, and $300 \mu M$ lapachol. (B, D, F) Survival assays were performed by treatment with sublethal and lethal doses and plating $100 \mu \mathrm{L}$ of serial dilutions onto LB agar plates after $4 \mathrm{~h}$ of stress exposure. The survival rates of CFUs for the treated samples were calculated relative to the control, which was set to $100 \%$. The $m h q R$ mutant is significantly more resistant to rifampicin and norfloxacin, which could be restored in the $m h q R$ complemented strain back to wild-type level. However, the $m h q R$ mutant is significantly more susceptible to the naphthoquinone lapachol than the wild type. The results are from four biological replicates. Error bars represent the standard deviation. ${ }^{*} p<0.05 ; *^{*} p<0.01 ; * * * p<0.001$.

mediates higher resistance to quinones, the $\mathrm{MhqR}$ regulon functions in resistance mechanisms against quinone-derived antimicrobials.

\section{The mhqR mutant shows differential susceptibilities to killing by murine macrophage in vivo and under oxidative stress in vitro}

To analyze the role of the $\mathrm{MhqR}$ regulon under infection conditions, we determined the survival of the $m h q R$ mutant in phagocytosis assays using the murine macrophage cell line J-774A.1, as previously described (44) (Fig. 8A, B). The colony-forming units (CFUs) of intracellular $S$. aureus were determined 2, 4, 24, and $48 \mathrm{~h}$ postinfection. At $24 \mathrm{~h}$ postinfection, the number of viable bacteria decreased to $\sim 20 \%$ for the wild type and $10 \%$ for the $m h q R$ mutant (Fig. 8A). Thus, the $m h q R$ mutant showed a $50 \%$ reduced survival rate compared with the wild type. This sensitive survival phenotype of the $m h q R$ mutant could be restored to $>90 \%$ in the $m h q R$ complemented strain (Fig. 8B). Interestingly, $48 \mathrm{~h}$ postinfection, the number of surviving bacteria increased to $\sim 20 \%$ for the $m h q R$ mutant and decreased to $6 \%$ for the wild type and $m h q R$ complemented strain (Fig. 8A, B). Thus, the intramacrophage survival of the $m h q R$ mutant was 2.5 -fold higher compared with the wild type after $48 \mathrm{~h}$ of infections (Fig. 8B). This indicates that the $m h q R$ mutation sensitizes $S$. aureus during early stages of macrophage infections, whereas improved survival of the $m h q R$ mutant is acquired during long-term infection inside macrophages.

Transcriptome analysis revealed that the peroxide-specific PerR regulon was downregulated in the $m h q R$ mutant under control and MHQ stress (Fig. 2 and Supplementary Fig. S2; Supplementary Table S2). Thus, we investigated the ROS susceptibility of the $m h q R$ mutant in vitro. Growth phenotype analyses revealed an increased susceptibility of the $m h q R$ deletion mutant under sublethal $1.5 \mathrm{~m} M \mathrm{NaOCl}$ and $10 \mathrm{~m} M$ hydrogen peroxide $\left(\mathrm{H}_{2} \mathrm{O}_{2}\right)$ stress (Fig. 8C, D). However, the $m h q R$ mutant showed an improved survival upon lethal $\mathrm{NaOCl}$ 

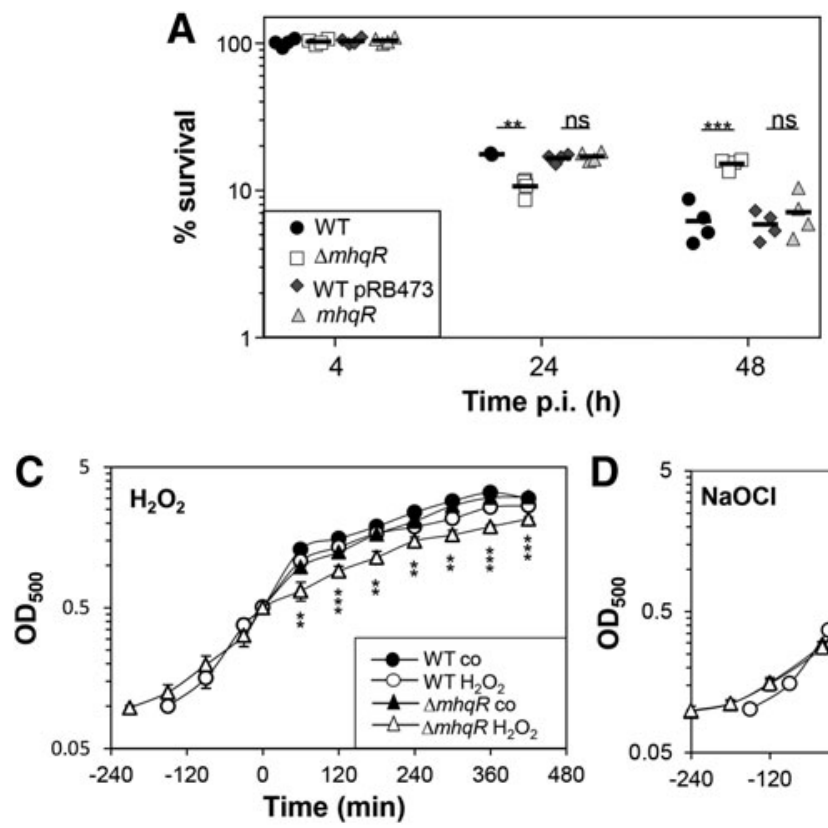

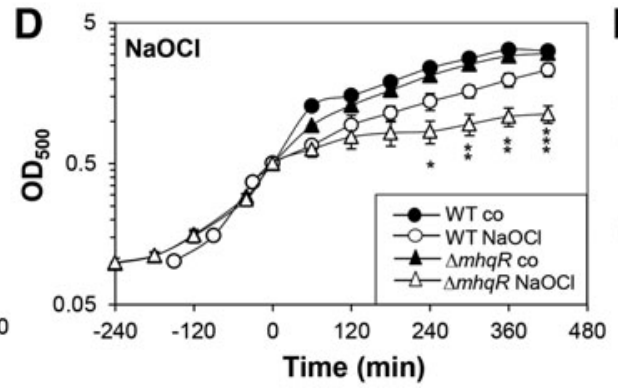

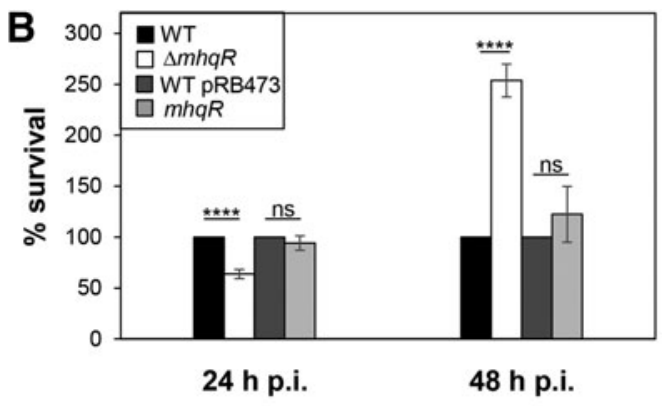

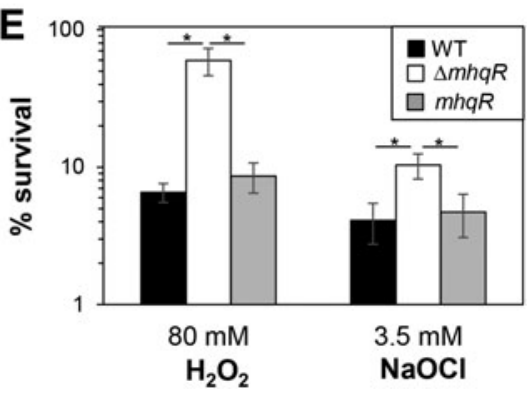

FIG. 8. The $m h q R$ mutant is impaired in survival inside J-774.1 murine macrophages after $24 \mathrm{~h}$ and growth sensitive under sublethal ROS and $\mathrm{NaOCl}$ but resistant to long-term infections and toxic $\mathrm{ROS}$ and $\mathrm{NaOCl}$. (A, B) The survival of S. aureus strains was analyzed 2, 4, 24, and $48 \mathrm{~h}$ postinfection (p.i.) of the murine macrophage cell line J-774A.1 and the CFUs were determined. (A) The percentages in survival of the wild type (WT), WT pRB473, $m h q R$ deletion mutant, and the $m h q R$ complemented strain $(m h q R)$ were calculated, and the survival at the $2 \mathrm{~h}$ time point was set to $100 \%$. (B) The average percentage in survival was calculated for each mutant and complemented strains in relation to the WT or WT pRB473, which was set to $100 \%$. Results of four biological replicates are presented as scatter dots in (A) and mean values (B). (C, D) For growth curves, $S$. aureus COL wild type, the $m h q R$ deletion mutant, and the $m h q R$ complemented strain

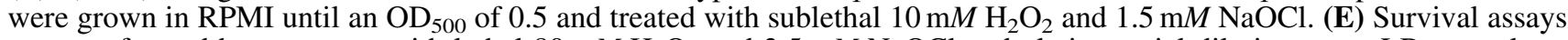
were performed by treatment with lethal $80 \mathrm{mM} \mathrm{H} \mathrm{H}_{2} \mathrm{O}_{2}$ and $3.5 \mathrm{mM} \mathrm{NaOCl}$ and plating serial dilutions onto LB agar plates after $4 \mathrm{~h}$ of stress exposure. The survival rates of CFUs for the treated samples were calculated relative to the control, which was set to $100 \%$. The results are from three biological replicates. Error bars represent the standard deviation. ns, $p>0.05$; $* p<0.05 ; * * p<0.01 ; * * *<<0.001 ; * * * * p \leq 0.0001 . \mathrm{H}_{2} \mathrm{O}_{2}$, hydrogen peroxide; ROS, reactive oxygen species.

and $\mathrm{H}_{2} \mathrm{O}_{2}$ stress compared with the wild type (Fig. 8E). The genetically encoded Brx-roGFP2 biosensor was applied to measure the changes in the $\mathrm{BSH}$ redox potential in the $m h q R$ mutant during the growth and under $\mathrm{H}_{2} \mathrm{O}_{2}$ stress (Supplementary Fig. S7). The basal level oxidation of the BrxroGFP2 was similar between the wild type and the $m h q R$ mutant. However, the $m h q R$ mutant showed a slightly higher oxidation increase and delayed recovery of the BSH redox potential compared with the wild type. Altogether, these results indicate that the $m h q R$ mutant is sensitive in growth to sublethal ROS and to the host immune defense during the first $24 \mathrm{~h}$ of macrophage infections. However, under long-term infection conditions $(48 \mathrm{~h})$ and lethal ROS concentrations, the MhqR regulon is an important defense mechanism and required for $S$. aureus survival, providing an attractive drug target.

\section{The mhqR mutant shows enhanced respiratory chain activity and increased ATP levels}

Quinones, such as menaquinone, are important electron carriers of the respiratory chain in S. aureus. Previous studies have shown that the quinone-sensing QsrR repressor responds also to menadione, the precursor of menaquinone in $S$. aureus (33). Thus, we investigated whether the upregulation of quinone degradation enzymes $\mathrm{MhqD}$ and $\mathrm{MhqE}$ in the $m h q R$ mutant affects the electron transport to reduce molecular oxygen in the respiratory chain. Oxygen consumption rates were measured using a Clark-type electrode for the $m h q R$ and $q s r R$ mutants during the exponential growth and stationary phases with $1 \mathrm{~m} M$ glucose or $100 \mathrm{~m} M$ succinate as electron donors (Fig. 9A).

During the exponential growth phase, all strains showed high oxygen consumption rates of $55-70 \mathrm{nmol} / \mathrm{mL} / \mathrm{min}$ with glucose as electron donor. The $m h q R$ mutant had a significantly increased oxygen consumption rate with glucose compared with the wild type, but no differences were observed with succinate. During the stationary phase, the oxygen consumption rate of the wild type was $\sim 35 \mathrm{nmol} / \mathrm{mL} /$ min with glucose, significantly increased in the $m h q R \mathrm{mu}-$ tant $(48 \mathrm{nmol} / \mathrm{mL} / \mathrm{min})$, but decreased in the $q s r R$ mutant (Fig. 9A). Similarly, stationary phase $m h q R$ mutant cells showed higher oxygen reduction rates with succinate $(28 \mathrm{nmol} / \mathrm{mL} / \mathrm{min})$. These results of the higher respiratory chain activity in the $m h q R$ mutant were also confirmed under microaerophilic conditions with methylene blue as indicator of oxygen consumption (Fig. 9B).

Due to the increased electron transport, elevated ATP levels could be determined in the $m h q R$ mutant compared with the wild type (Fig. 9C). Thus, we speculate that quinones are more reduced in the $m h q R$ mutant leading to an increased electron transport and higher ATP levels, which is supported 

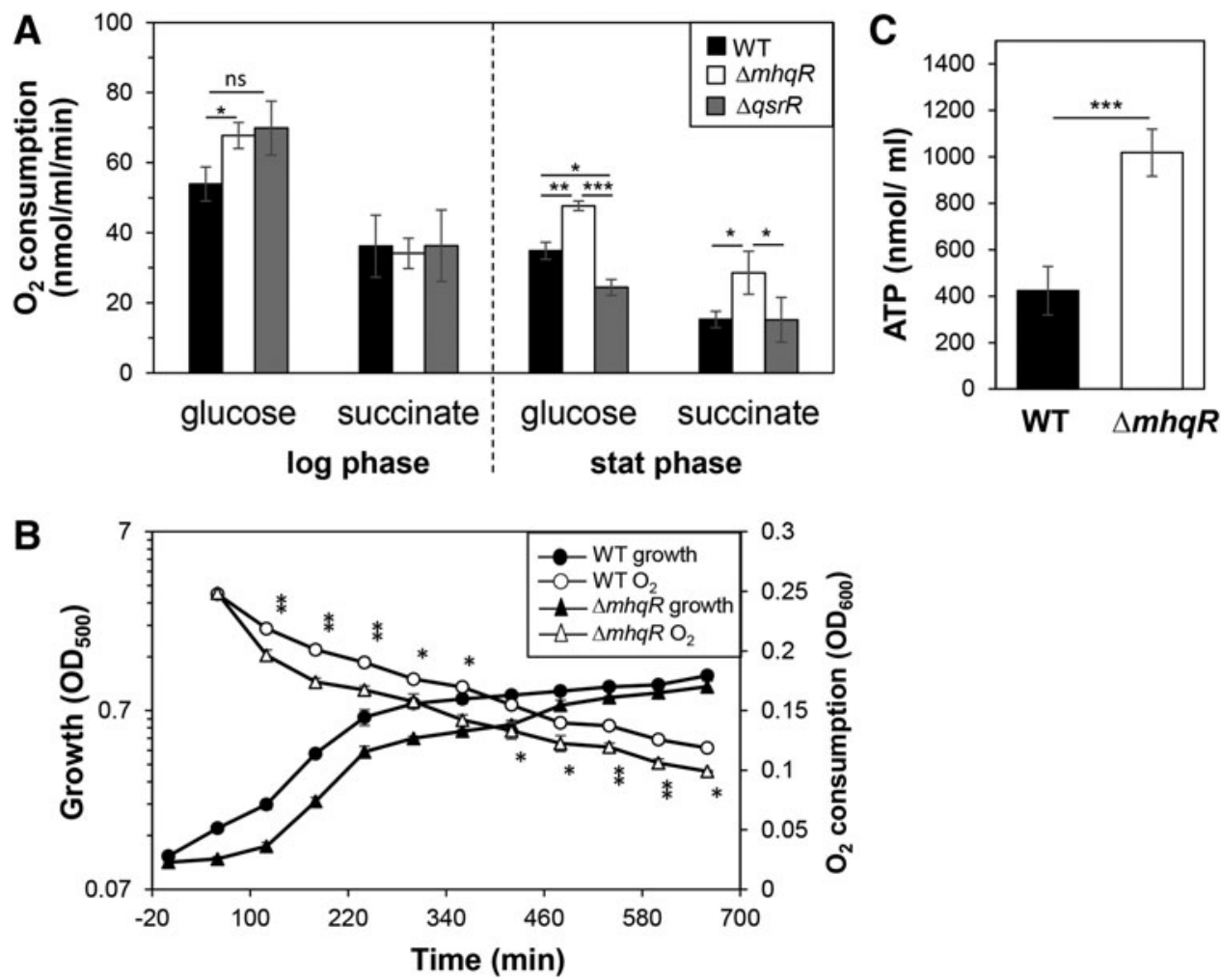

FIG. 9. The $m h q R$ mutant shows a higher respiratory chain activity and increased ATP level. (A) Oxygen consumption rates of the wild type and $m h q R$ and $q s r R$ mutants were determined during the exponential growth and stationary phases with glucose or succinate as electron donor using a Clark-type electrode. The results are presented as average values of three biological replicates with standard deviations. (B) To measure oxygen consumption under microaerophilic conditions, discoloration of methylene blue was measured as absorbance change at $\mathrm{OD}_{600}$ together with the $\mathrm{OD}_{500}$ as bacterial growth. (C) The ATP levels of the wild type and the $m h q R$ mutant were determined during the exponential growth phrase with the ATP Bioluminescence Assay Kit CLS II (Sigma-Aldrich) according to the manufacturer's instructions. The results are from four biological replicates. Error bars represent the standard deviation. ns, $p>0.05 ; * p<0.05 ; * * p<0.01 ; * * * p<0.001$.

by reduced expression of oxidative stress-specific genes in the transcriptome of the $m h q R$ mutant.

\section{Discussion}

In this study, we characterized the novel quinone-sensing MhqR repressor of $S$. aureus as an important component of the global response of $S$. aureus to quinones and antimicrobials. Transcriptome analysis in response to MHQ revealed the global signature of a thiol-specific oxidative and electrophile stress response, which is evident by the induction of the PerR, QsrR, MhqR, CtsR, and HrcA regulons. In addition, quinones caused a metal, sulfide, and cell wall stress response by upregulation of the Fur, CsoR, CstR, and GraRS regulons. This transcriptome profile overlaps strongly with the response to quinones in B. subtilis as shown by the inductions of the PerR, Spx, YodB, MhqR, HrcA, and CtsR regulons $(1,29,42,55,68,69)$.

The MhqR and QsrR regulons represent the quinone stress signature in $S$. aureus. The QsrR regulon includes genes encoding ring-cleavage dioxygenases (catE, catE2), quinone reductases (azoRl, frp), and nitroreductases (yodC) (33). The MhqR regulon consists only of the $m h q R E D$ operon in $S$. aureus (Fig. 10). MhqD is annotated as phospholipase/carboxylesterase of the widespread alpha/beta fold hydrolase family
(59). These enzymes cleave carboxylate esters to acids and alcohols and might be involved in the catabolism of quinone compounds. MhqE encodes a ring-cleavage dioxygenase in $S$. aureus. Thus, paralogous ring-cleavage dioxygenases and the nitro- and quinone reductases confer additive resistance to MHQ in S. aureus. Homologous dioxygenases (CatE, MhqA, MhqE, MhqO), quinone, and nitroreductases (AzoR1, AzoR2, YodC) have been shown to function in detoxification of exogenous quinones and catecholic compounds (Fig. 10) (12, 13, $42,55,68,69)$, as well as the endogenous catecholate siderophore bacillibactin in $B$. subtilis (65). Thus, the QsrR and MhqR regulons have a similar composition of detoxification genes in both bacteria and confer resistance to quinones.

The catechol-2,3-dioxygenases CatE of B. subtilis was previously shown to cleave catechol to produce 2hydroxymuconic semialdehyde $(55,68)$, whereas the dioxygenase MhqE of the MhqR regulon shares strong homology to hydroquinone-type 1,2-dioxygenase LinE of Sphingomonas paucimobilis that is involved in degradation of the xenobiotic insecticide hexachlorocyclohexane (51). Catechol-2,3dioxygenases are iron-containing enzymes (51), and CatE was shown to respond also to iron limitation in B. subtilis through control by the Fur repressor (65). Thus, the $S$. aureus dioxygenases could be also involved in the decomposition of siderophores. S. aureus utilizes carboxylate siderophores 


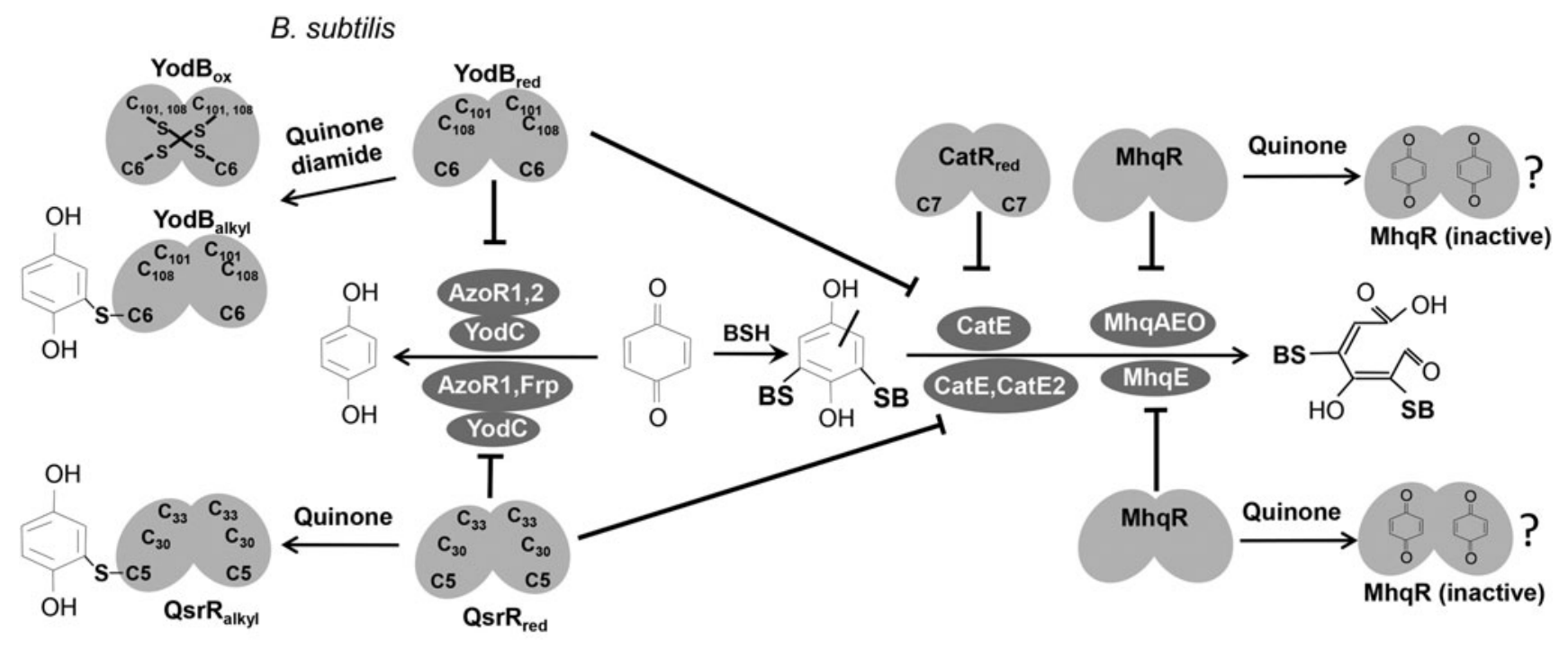

S. aureus

FIG. 10. The roles of the quinone-sensing MhqR, CatR, and YodB/QsrR regulons in B. subtilis and $S$. aureus. Exposure of B. subtilis and $S$. aureus to quinones induces the quinone detoxification regulons controlled by the homologous MarR-type repressors MhqR, YodB/QsrR, and CatR. The redox-sensing MarR/DUF24-family repressors YodB and CatR of B. subtilis are inactivated by intersubunit disulfide formation in vivo that involves the conserved Cys6 or Cys7 $(1,2,12,13$, 69). The YodB and QsrR repressor mutant proteins with single Cys6 and Cys5 sense quinones also by thiol-S-alkylation in vitro $(33,41)$. The MhqR repressors might be inactivated by direct binding of quinones to a specific pocket. The MhqR and YodB/QsrR regulon include homologous quinone reductases, nitroreductases, and dioxygenases for quinone and diamide detoxification. The thiol-dependent dioxygenases $\mathrm{MhqA}$, MhqE, CatE, and $\mathrm{MhqO}$ of $B$. subtilis and their respective homologs CatE, MhqE, and CatE2 of S. aureus (Supplementary Fig. S3B) are involved in ring cleavage of quinone-Sadducts. The quinone reductases AzoR1 and AzoR2 of B. subtilis and AzoR1 and Frp of S. aureus and the nitroreductases YodC and MhqN of B. subtilis and YodC of $S$. aureus catalyze the reduction of quinones to redox stable hydroquinones.

staphyloferrin A and B but can also import xenosiderophores of other bacteria (25). However, the S. aureus $m h q R$ and $q s r R$ mutants showed no growth and survival phenotype upon treatment with the iron-scavenger 2,2'-dipyridyl compared with the wild type, indicating no function under iron limitation (Supplementary Fig. S8). More detailed studies are required to define the precise functions of the many detoxification enzymes of the MhqR and QsrR regulons in S. aureus.

MhqR belongs to the widespread MarR family of transcriptional regulators harboring wHTH DNA binding motifs that bind to 16-20 bp (pseudo) palindromic double-stranded DNA in adjacent major grooves (16). In previous studies, we identified a conserved 9-9 bp inverted repeat sequence as MhqR operator site for B. subtilis MhqR (69). This palindromic operator sequence was conserved in the $S$. aureus mhqRED upstream promoter region.

DNA binding assays revealed specific binding of MhqR to its operator with a high affinity $\left(K_{\mathrm{d}}=7.38 \mathrm{n} M\right)$. Comparative studies have shown that the dissociation constants vary across MarR type regulators $(37,75)$. However, the $K_{\mathrm{d}}$ value of MhqR is in the range of other MarR-type regulators, such as OhrR of B. subtilis $\left(K_{\mathrm{d}}=5 \mathrm{n} M\right)$ and MepR of $S$. aureus $\left(K_{\mathrm{d}}=6.3 \mathrm{n} M\right)(22,36)$.

DNA binding assays further revealed that quinones lead to inhibition of the DNA binding activity of MhqR, which does not involve a thiol-based mechanism. Cys 95 of MhqR is also not conserved in other MhqR homologs and dispensable for quinone regulation and DNA binding in vivo and in vitro. No involvement of the nonconserved Cys126 in quinone regulation was also shown for the B. subtilis MhqR protein (69).
Thus, the regulatory mechanism of MhqR is different compared with redox-sensing MarR-type or Rrf2-family regulators, which sense directly redox-active compounds, such as ROS, hypochlorous acid ( $\mathrm{HOCl})$, or quinones by specific conserved redox-sensitive Cys residues $(2,29,44)$. These redox-sensing regulators include YodB, CatR, HypR, and OhrR of B. subtilis and their homologs QsrR, SarZ, and MgrA of $S$. aureus $(11,12,29,33,41,42,44,60,61)$.

We speculate that the quinone-sensing mechanism of MhqR occurs via a direct binding of the quinone as ligand to a specific pocket. The DNA binding activity of many MarR-type regulators is altered by chemical ligands, such as phenolic or aromatic compounds (e.g., salicylate, urate, protocatechuate, hydroxyphenylacetate, $p$-hydroxycinnamateCoA) $(24,62,75)$. Structural and biochemical studies of ligand-binding MarR-family proteins suggest a shared ligandbinding pocket between dimerization and DNA binding regions (16). This common ligand-binding pocket was also identified between the dimer interface and the wHTH motif in the structure of the MarR-type regulator ST1710 of Sulfolobus tokodaii in complex with its inhibitor salicylate (38).

The structure of the ST1710-salicylate complex was used as template to model the MhqR structure of S. aureus using SWISS-MODEL (Supplementary Fig. S5A). However, the salicylate contact residues Tyr37 and Tyr111 of one subunit and Ala16, Lys17, and Arg20 of the opposing subunit in the ST1710 dimer are not conserved in MhqR of S. aureus. Thus, the specific interactions of the putative ligand-binding pocket of MhqR with quinones and the resulting conformational changes in the wHTH motifs remain to be elucidated. 
Apart from quinone resistance, the MhqR regulon also confers broad-spectrum antimicrobial resistance to quinonelike compounds in $S$. aureus, such as pyocyanin, ciprofloxacin, norfloxacin, and rifampicin. The fluoroquinolones ciprofloxacin and norfloxacin are priority class antibiotics to combat $S$. aureus infections, which act as DNA gyrase and topoisomerase inhibitors, causing superoxide anions and hydroxyl radicals through gyrase poisoning $(19,64)$. Pyocyanin is produced by Pseudomonas aeruginosa, a pathogen often co-isolated with $S$. aureus in cystic fibrosis patients. Pyocyanin blocks the electron transport chain by trapping electrons from NADH $(26,58)$. Mutations in $q s r R$ have been previously selected as pyocyanin resistance mechanism (56). Rifampicin inhibits the RNA polymerase resulting in frequent $r p o B$ mutations as resistance mechanism in S. aureus (74).

Our study revealed an involvement of the MhqR and QsrR regulons in antimicrobial resistance toward quinone-like antimicrobials in S. aureus. Similarly, the MarR-type regulators MarR of Escherichia coli, MgrA and MepR of S. aureus, as well as MexR in $P$. aeruginosa have been shown to confer resistance to multiple antibiotics by controlling efflux pumps $(10,14,64,70)$. We hypothesize that the MhqR- and QsrR-controlled dioxygenases and quinone reductases contribute to detoxification of the antimicrobial compounds with quinone structures as new resistance mechanism. There is also the controversial debate about the involvement of ROS generation in the killing mode of antibiotics. Thus, the antibiotic resistant phenotypes of the $m h q R$ mutant could be connected to its ROS resistance in survival assays.

However, the MhqR regulon did not confer resistance to the naphthoquinone lapachol. Differences in the detoxification of benzoquinones and naphthoquinones have been described in E. coli (77). In S. aureus, flavohemoglobin has high substrate specificity for 2-hydroxy-1,4-naphthoquinones and might be more specific for naphthoquinone detoxification (53).

While the MhqR regulon plays an important role in antibiotic resistance, the $m h q R$ mutant showed increased sensitivity at early time points of $24 \mathrm{~h}$ after macrophage infections and under sublethal ROS and $\mathrm{HOCl}$ exposure in vitro. We hypothesize that the lower basal transcription of PerR regulon genes in the $m h q R$ mutant could contribute to the $\mathrm{H}_{2} \mathrm{O}_{2-}$ and $\mathrm{NaOCl}$-sensitive phenotypes as well as to decreased survival in infection assays. Surprisingly, the $m h q R$ was delayed in growth after sublethal $\mathrm{HOCl}$ and $\mathrm{H}_{2} \mathrm{O}_{2}$ but acquired resistance to lethal doses of $\mathrm{NaOCl}$ and $\mathrm{H}_{2} \mathrm{O}_{2}$ in killing assays. In addition, at a later time point, $48 \mathrm{~h}$ postinfection of macrophages, the $m h q R$ mutant showed a higher survival rate than the wild type.

It could be possible that the respiratory chain activity is decreased in the $S$. aureus $m h q R$ mutant, as has been proposed in the B. subtilis $m h q R$ mutant (34). Decreased respiratory chain activity was linked to lower ROS levels and facilitated growth of antibiotic resistant cell wall-deficient Lforms in B. subtilis (34). The qsrR mutant indeed showed decreased oxygen consumption with glucose, but only during the stationary phase. However, the $m h q R$ mutant had a higher respiratory chain activity and increased ATP levels than the wild type. Thus, it might be possible that quinones are more reduced in the $m h q R$ mutant, leading to enhanced electron transport. Our future analyses are directed to further investigate the functions and redox-sensing mechanisms of MhqR and QsrR in response to quinones and related antimicrobials.

\section{Experimental Procedures}

\section{Bacterial strains, growth, and survival assays}

Bacterial strains, plasmids, and primers are listed in Supplementary Tables S3 and S4. E. coli was cultivated in LuriaBertani (LB) broth medium and $S$. aureus in RPMI medium. Survival assays were performed by plating $100 \mu \mathrm{L}$ of serial dilutions of $S$. aureus onto LB agar plates and determination of CFUs. Statistical analysis was performed using Student's unpaired two-tailed $t$-test by the graph prism software. The compounds used for growth and survival assays (e.g., MHQ, ciprofloxacin, norfloxacin, lapachol, pyocyanin, $\mathrm{H}_{2} \mathrm{O}_{2}, \mathrm{NaOCl}$ ) were purchased from Sigma-Aldrich. $\mathrm{NaOCl}$ dissociates in aqueous solution to $\mathrm{HOCl}$ and hypochlorite $\left(\mathrm{OCl}^{-}\right)$(20). The concentration of $\mathrm{HOCl}$ was determined by absorbance measurements, as reported previously (76).

\section{Construction of the $\mathrm{S}$. aureus $\mathrm{COL} \mathrm{mhqR}$ and qsrR deletion mutants and the complemented mhqR and mhqRC95A mutant strains}

The S. aureus COL mhqR (SACOL2531) and qsrR (SACOL2115) deletion mutants were constructed by allelic replacement via pMAD, as described previously $(4,44)$. The $500 \mathrm{bp}$ upstream and downstream regions of $m h q R$ and $q s r R$ were each fused by overlap extension PCR and ligated into the $B g l I I$ and SalI sites of plasmid pMAD. The pMAD constructs were electroporated into $S$. aureus RN4220, transferred to $S$. aureus COL by phage transduction, and selected for plasmid excision leading to clean deletions of $m h q R$ and $q s r R$, as described previously $(44,66)$.

The complemented $m h q R$ and $m h q R C 95 A$ mutant strains were constructed using the pRB473 plasmid, as described previously (44). The $m h q R$ and $m h q R C 95 A$ sequences were amplified from plasmids pET11b-mhqR and pET11b$m h q R C 95 A$, digested with BamHI and KpnI, and inserted into pRB473 resulting in plasmids pRB473-mhqR and pRB473$m h q R C 95 A$ (Supplementary Table S3). The plasmids were introduced into the $m h q R$ mutant via phage transduction, as described previously (44).

\section{RNA isolation, Northern blot analysis, \\ RNA-seq transcriptomics, and bioinformatics}

For RNA isolation, $S$. aureus COL was cultivated in RPMI medium and treated with $45 \mu M \mathrm{MHQ}, 300 \mu M$ lapachol, $90.5 \mu M$ ciprofloxacin, $76 \mu M$ pyocyanin, $1 \mathrm{~m} M \mathrm{NaOCl}$, $0.5 \mathrm{~m} M$ methylglyoxal, $2 \mathrm{~m} M$ diamide, and $0.75 \mathrm{~m} M$ formaldehyde for 15 and $30 \mathrm{~min}$, as described previously (73). Northern blot hybridizations were performed with the digoxigenin-labeled $m h q D$-specific antisense RNA probe synthesized in vitro using T7 RNA polymerase and the primer pairs SACOL2529-for/rev (Supplementary Table S4), as described previously $(68,73)$.

RNA-seq transcriptomics was performed using RNA of $S$. aureus $\mathrm{COL}$ and the $m h q R$ deletion mutant isolated before and $30 \mathrm{~min}$ after $45 \mu M \mathrm{MHQ}$, as described in previous studies (72). Differential gene expression analysis of three biological replicates was performed using DESeq2 (46) with ReadXplorer v2.2 (28) as described previously (72) using an adjusted $p$-value cutoff of $\leq 0.05$ and a signal intensity ratio ( $M$-value) cutoff of $\geq 0.6$ or $\leq-0.6$ (fold-change of \pm 1.5$)$. 
The cDNAs enriched for primary $5^{\prime}$-transcripts were prepared according to the method described previously (63). cDNAs were sequenced paired end on an Illumina MiSeq System (San Diego, CA) using 75 bp read length. The R1 cDNA reads were mapped to the $S$. aureus USA300 TCH1516 genome (27) with bowtie2 v2.2.7 (40) using the default settings for single-end read mapping and visualized with Read Explorer v.2.2 (28). The whole transcriptome and $5^{\prime}$ enriched RNA-seq raw data files are available in the ArrayExpress database under E-MTAB-7074 and E-MTAB7385.

Cloning, expression, and purification of His-tagged MhqR and MhqRC95A mutant protein in $\mathrm{E}$. coli

The $m h q R$ gene (SACOL2531) was amplified from chromosomal DNA of $S$. aureus COL by PCR using primers SACOL2531-pET-for-NheI and SACOL2531-pET-revBamHI (Supplementary Table S4), digested with NheI and BamHI, and inserted into plasmid pET11b (Novagen) to generate plasmid pET11b-mhqR. For the construction of $m h q R C 95 A$ mutant, two first-round PCRs were performed using primer pairs SACOL2531-pET-for-NheI and SACOL2531-pET-C95A-Rev as well as primer pairs SACOL2531-pET-C95A-for and SACOL2531-pET-revBamHI (Supplementary Table S4). The two first-round PCR products were hybridized and amplified by a second round of PCR using primers SACOL2531-pET-for-NheI and SACOL2531-pET-rev-BamHI. The second-round PCR products were digested with NheI and BamHI and inserted into plasmid pET11b to generate plasmid pET11b-mhqRC95A. For expression and purification of His-tagged MhqR and MhqRC95A proteins, E. coli BL21(DE3) plysS was used with the plasmids pET11b-mhqR and pET11b-mhqRC95A, as described previously (44). Cultivation of the E. coli expression strains was performed in $1 \mathrm{~L} \mathrm{LB}$ medium until the exponential growth phase at $\mathrm{OD}_{600}$ of 0.8 , followed by the addition of $1 \mathrm{~m} M$ isopropyl- $\beta$-D-thiogalactopyranoside for $5 \mathrm{~h}$ at $30^{\circ} \mathrm{C}$. Recombinant His-tagged MhqR and the MhqRC95A mutant proteins were purified, as described previously (44).

\section{EMSAs of MhqR and MhqRC95A proteins}

For EMSAs, the DNA fragment containing the $m h q R$ upstream region was amplified by PCR with the primer set emsa2531-for and emsa2531-rev (Supplementary Table S4). The DNA-binding reactions were performed with $15 \mathrm{ng} / \mu \mathrm{L}$ PCR product and purified His-MhqR and His-MhqRC95A proteins for $45 \mathrm{~min}$, as described previously (44). MHQ was added to the DNA-MhqR-complex for $30 \mathrm{~min}$ to observe the dissociation of MhqR from the DNA. To analyze the reversibility of inhibition of MhqR by quinones, DTT was added $30 \mathrm{~min}$ after MHQ addition to the MhqR-DNA reaction. Thus, MHQ and DTT were added subsequently to the DNA-MhqR-complex for each $30 \mathrm{~min}$. EMSAs were carried out as described previously (44).

\section{Brx-roGFP2 biosensor measurements}

$S$. aureus COL and mhqR mutant strains with the BrxroGFP2 biosensor plasmids were cultivated in LB and used for measurements of the biosensor oxidation degree along the growth curves and after injection of $\mathrm{H}_{2} \mathrm{O}_{2}$, as described previously (45). Fully reduced and oxidized controls were treated with $10 \mathrm{~m} M$ DTT and $5 \mathrm{~m} M$ diamide or $20 \mathrm{~m} M$ cumene hydroperoxide, respectively. Brx-roGFP2 biosensor fluorescence emission was measured at $510 \mathrm{~nm}$ after excitation at 405 and $488 \mathrm{~nm}$ using the CLARIOstar Microplate Reader (BMG Labtech), as described previously (45).

\section{Macrophage infection assays}

The infection assays were performed using the murine macrophage cell line J-774A.1, as described previously (44). Intracellular survival of phagocytosed $S$. aureus was measured after 2, 4, 24, and $48 \mathrm{~h}$ postinfection by determination of CFUs, as described previously (44).

\section{Determination of oxygen consumption rates}

The oxygen consumption rates of $S$. aureus strains were determined with a Clark-type electrode (Oxygraph; Hansatech) at $25^{\circ} \mathrm{C}$ according to a modified protocol, as described previously $(50,78)$. For determination of the respiratory chain activity during the exponential growth and stationary phases, cells were grown in tryptic soy broth medium to an $\mathrm{OD}_{600}$ of 0.6 and for $24 \mathrm{~h}$. Cells were harvested by centrifugation, washed in $33 \mathrm{~m} M$ potassium phosphate buffer $(\mathrm{pH}$ 7.0), and adjusted to an $\mathrm{OD}_{578}$ of 5. Oxygen consumption was measured upon addition of $100 \mathrm{~m} M$ disodium succinate or $1 \mathrm{~m} M$ glucose as electron donors in three bioreplicates. Measurements were corrected for basal oxygen consumption without electron donors.

In addition, colorimetric determination of the oxygen consumption rates was performed by discoloration of methylene blue. Methylene blue was added at a final concentration of $0.004 \mathrm{mg} / \mathrm{mL}$ to $40 \mathrm{~mL}$ of $S$. aureus cells that were cultivated under microaerophilic conditions. The discoloration of methylene blue was determined as absorbance change at $\mathrm{OD}_{600}$ together with the optical density of the culture at $\mathrm{OD}_{500}$.

\section{ATP measurements}

The ATP levels of $S$. aureus strains were determined with the ATP Bioluminescence Assay Kit CLS II (Sigma-Aldrich) according to the manufacturer's instructions. Briefly, $1 \mathrm{~mL}$ of exponentially growing cells was harvested, resuspended in $100 \mu \mathrm{L}$ dilution buffer, and disrupted by boiling in $900 \mu \mathrm{L}$ of $100 \mathrm{~m} M$ Tris, $4 \mathrm{~m} M$ ethylenediaminetetraacetic acid, $\mathrm{pH} 7.75$, for $2 \mathrm{~min}$. After centrifugation of the lysate, $50 \mu \mathrm{L}$ of the supernatant was incubated with $50 \mu \mathrm{L}$ luciferase and the luminescence was measured using the CLARIOstar Microplate Reader (BMG Labtech). The values were corrected for the autoluminescence of the cells, and the ATP level was determined based on the ATP standard curve.

\section{Acknowledgments}

We thank Prof. Holger Dau (Department of Physics, Freie Universität Berlin) for providing the Clark electrode. This work was supported by an ERC Consolidator grant (GA 615585) MYCOTHIOLOME and grants from the Deutsche Forschungsgemeinschaft (AN746/4-1 and AN746/4-2) within the SPP1710, by the SFB973 project C08N, and by the SFB/TR84 project B06 to H.A. 


\section{Author Disclosure Statement}

No competing financial interests exist.

\section{Supplementary Material}

Supplementary Table S1

Supplementary Table S2

Supplementary Table S3

Supplementary Table S4

Supplementary Figure S1

Supplementary Figure S2

Supplementary Figure S3

Supplementary Figure S4

Supplementary Figure S5

Supplementary Figure S6

Supplementary Figure S7

Supplementary Figure S8

\section{References}

1. Antelmann $\mathrm{H}$, Hecker M, and Zuber P. Proteomic signatures uncover thiol-specific electrophile resistance mechanisms in Bacillus subtilis. Expert Rev Proteomics 5: 77-90, 2008.

2. Antelmann $\mathrm{H}$ and Helmann JD. Thiol-based redox switches and gene regulation. Antioxid Redox Signal 14: 1049-1063, 2011.

3. Archer GL. Staphylococcus aureus: a well-armed pathogen. Clin Infect Dis 26: 1179-1181, 1998.

4. Arnaud M, Chastanet A, and Débarbouillé M. New vector for efficient allelic replacement in naturally nontransformable, low-GC-content, gram-positive bacteria. Appl Environ Microbiol 70: 6887-6891, 2004.

5. Baker J, Sengupta M, Jayaswal RK, and Morrissey JA. The Staphylococcus aureus CsoR regulates both chromosomal and plasmid-encoded copper resistance mechanisms. Environ Microbiol 13: 2495-2507, 2011.

6. Biasini M, Bienert S, Waterhouse A, Arnold K, Studer G, Schmidt T, Kiefer F, Gallo Cassarino T, Bertoni M, Bordoli L, and Schwede T. SWISS-MODEL: modelling protein tertiary and quaternary structure using evolutionary information. Nucleic Acids Res 42: W252-W258, 2014.

7. Bittner S. When quinones meet amino acids: chemical, physical and biological consequences. Amino Acids 30: 205-224, 2006.

8. Boucher HW and Corey GR. Epidemiology of methicillinresistant Staphylococcus aureus. Clin Infect Dis 46 Suppl 5: S344-S349, 2008.

9. Chandrangsu P, Loi VV, Antelmann H, and Helmann JD. The role of bacillithiol in Gram-positive Firmicutes. Antioxid Redox Signal 28: 445-462, 2018.

10. Chen H, Hu J, Chen PR, Lan L, Li Z, Hicks LM, Dinner $\mathrm{AR}$, and He C. The Pseudomonas aeruginosa multidrug efflux regulator MexR uses an oxidation-sensing mechanism. Proc Natl Acad Sci U S A 105: 13586-13591, 2008.

11. Chen PR, Nishida S, Poor CB, Cheng A, Bae T, Kuechenmeister L, Dunman PM, Missiakas D, and He C. A new oxidative sensing and regulation pathway mediated by the MgrA homologue SarZ in Staphylococcus aureus. Mol Microbiol 71: 198-211, 2009.

12. Chi BK, Albrecht D, Gronau K, Becher D, Hecker M, and Antelmann $\mathrm{H}$. The redox-sensing regulator YodB senses quinones and diamide via a thiol-disulfide switch in $\mathrm{Ba}$ cillus subtilis. Proteomics 10: 3155-3164, 2010.
13. Chi BK, Kobayashi K, Albrecht D, Hecker M, and Antelmann H. The paralogous MarR/DUF24-family repressors YodB and CatR control expression of the catechol dioxygenase CatE in Bacillus subtilis. J Bacteriol 192: 45714581, 2010.

14. Cohen SP, McMurry LM, Hooper DC, Wolfson JS, and Levy SB. Cross-resistance to fluoroquinolones in multipleantibiotic-resistant (Mar) Escherichia coli selected by tetracycline or chloramphenicol: decreased drug accumulation associated with membrane changes in addition to $\mathrm{OmpF}$ reduction. Antimicrob Agents Chemother 33: 1318-1325, 1989.

15. Cosgrove SE, Sakoulas G, Perencevich EN, Schwaber MJ, Karchmer AW, and Carmeli Y. Comparison of mortality associated with methicillin-resistant and methicillinsusceptible Staphylococcus aureus bacteremia: a metaanalysis. Clin Infect Dis 36: 53-59, 2003.

16. Deochand DK and Grove A. MarR family transcription factors: dynamic variations on a common scaffold. Crit Rev Biochem Mol Biol 52: 595-613, 2017.

17. Domingue GJ, Sr., and Woody HB. Bacterial persistence and expression of disease. Clin Microbiol Rev 10: 320-344, 1997.

18. Duy NV, Mäder U, Tran NP, Cavin JF, Tam le T, Albrecht $\mathrm{D}$, Hecker M, and Antelmann $\mathrm{H}$. The proteome and transcriptome analysis of Bacillus subtilis in response to salicylic acid. Proteomics 7: 698-710, 2007.

19. Dwyer DJ, Kohanski MA, Hayete B, and Collins JJ. Gyrase inhibitors induce an oxidative damage cellular death pathway in Escherichia coli. Mol Syst Biol 3: 91, 2007.

20. Estrela C, Estrela CRA, Barbin EL, Spanó JCE, Marchesan MA, and Pécora JD. Mechanism of action of sodium hypochlorite. Braz Dent J 13: 113-117, 2002.

21. Falord M, Mäder U, Hiron A, Debarbouille M, and Msadek T. Investigation of the Staphylococcus aureus GraSR regulon reveals novel links to virulence, stress response and cell wall signal transduction pathways. PLoS One 6: e21323, 2011.

22. Fuangthong $M$ and Helmann JD. The OhrR repressor senses organic hydroperoxides by reversible formation of a cysteine-sulfenic acid derivative. Proc Natl Acad Sci U S A 99: 6690-6695, 2002.

23. Gaupp R, Ledala N, and Somerville GA. Staphylococcal response to oxidative stress. Front Cell Infect Microbiol 2: 33, 2012.

24. Grove A. Regulation of metabolic pathways by MarR family transcription factors. Comput Struct Biotechnol J 15: 366-371, 2017.

25. Hammer ND and Skaar EP. Molecular mechanisms of Staphylococcus aureus iron acquisition. Annu Rev Microbiol 65: 129-147, 2011.

26. Hassan HM and Fridovich I. Mechanism of the antibiotic action pyocyanine. J Bacteriol 141: 156-163, 1980.

27. Highlander SK, Hulten KG, Qin X, Jiang H, Yerrapragada S, Mason EO, Jr., Shang Y, Williams TM, Fortunov RM, Liu Y, Igboeli O, Petrosino J, Tirumalai M, Uzman A, Fox GE, Cardenas AM, Muzny DM, Hemphill L, Ding Y, Dugan S, Blyth PR, Buhay CJ, Dinh HH, Hawes AC, Holder M, Kovar CL, Lee SL, Liu W, Nazareth LV, Wang Q, Zhou J, Kaplan SL, and Weinstock GM. Subtle genetic changes enhance virulence of methicillin resistant and sensitive Staphylococcus aureus. BMC Microbiol 7: 99, 2007.

28. Hilker R, Stadermann KB, Schwengers O, Anisiforov E, Jaenicke S, Weisshaar B, Zimmermann T, and Goesmann 
A. ReadXplorer 2-detailed read mapping analysis and visualization from one single source. Bioinformatics 32: 3702-3708, 2016.

29. Hillion M and Antelmann H. Thiol-based redox switches in prokaryotes. Biol Chem 396: 415-444, 2015.

30. Hillion M, Bernhardt J, Busche T, Rossius M, Maass S, Becher D, Rawat M, Wirtz M, Hell R, Rückert C, Kalinowski $\mathrm{J}$, and Antelmann $\mathrm{H}$. Monitoring global protein thiol-oxidation and protein S-mycothiolation in Mycobacterium smegmatis under hypochlorite stress. Sci Rep 7: 1195, 2017.

31. Hoffmann-Ostenhof O. Enzyme Inhibition by Quinones Metabolic Inhibitors V2: A Comprehensive Treatise. Burlington, VT: Elsevier Science, 1963.

32. Hussain H and Green IR. Lapachol and lapachone analogs: a journey of two decades of patent research(1997-2016). Expert Opin Ther Pat 27: 1111-1121, 2017.

33. Ji Q, Zhang L, Jones MB, Sun F, Deng X, Liang H, Cho H, Brugarolas P, Gao YN, Peterson SN, Lan L, Bae T, and He C. Molecular mechanism of quinone signaling mediated through S-quinonization of a YodB family repressor QsrR. Proc Natl Acad Sci U S A 110: 5010-5015, 2013.

34. Kawai Y, Mercier R, Wu LJ, Dominguez-Cuevas P, Oshima T, and Errington J. Cell growth of wall-free L-form bacteria is limited by oxidative damage. Curr Biol 25: 1613-1618, 2015.

35. Kumagai Y, Koide S, Taguchi K, Endo A, Nakai Y, Yoshikawa T, and Shimojo N. Oxidation of proximal protein sulfhydryls by phenanthraquinone, a component of diesel exhaust particles. Chem Res Toxicol 15: 483-489, 2002.

36. Kumaraswami M, Schuman JT, Seo SM, Kaatz GW, and Brennan RG. Structural and biochemical characterization of MepR, a multidrug binding transcription regulator of the Staphylococcus aureus multidrug efflux pump MepA. Nucleic Acids Res 37: 1211-1224, 2009.

37. Kumarevel T. The MarR family of transcriptional regulatorsa structural perspective. In: Pana M (ed.). Antibiotic Resistant Bacteria-A Continuous Challenge in the New Millennium. London, United Kingdom: IntechOpen, 2012, pp. 403-418.

38. Kumarevel T, Tanaka T, Umehara T, and Yokoyama S. ST1710-DNA complex crystal structure reveals the DNA binding mechanism of the MarR family of regulators. $\mathrm{Nu}$ cleic Acids Res 37: 4723-4735, 2009.

39. Kurosu M, Begari E. Vitamin K2 in electron transport system: are enzymes involved in vitamin K2 biosynthesis promising drug targets? Molecules 15: 1531-1553, 2010.

40. Langmead B and Salzberg SL. Fast gapped-read alignment with Bowtie 2. Nat Methods 9: 357-359, 2012.

41. Lee SJ, Lee IG, Lee KY, Kim DG, Eun HJ, Yoon HJ, Chae S, Song SH, Kang SO, Seo MD, Kim HS, Park SJ, and Lee BJ. Two distinct mechanisms of transcriptional regulation by the redox sensor YodB. Proc Natl Acad Sci U S A 113: E5202-E5211, 2016.

42. Leelakriangsak M, Huyen NT, Töwe S, van Duy N, Becher D, Hecker M, Antelmann H, and Zuber P. Regulation of quinone detoxification by the thiol stress sensing DUF24/ MarR-like repressor, YodB in Bacillus subtilis. Mol Microbiol 67: 1108-1124, 2008.

43. Liebeke M, Pöther DC, van Duy N, Albrecht D, Becher D, Hochgräfe F, Lalk M, Hecker M, and Antelmann H. Depletion of thiol-containing proteins in response to quinones in Bacillus subtilis. Mol Microbiol 69: 1513-1529, 2008.

44. Loi VV, Busche T, Tedin K, Bernhardt J, Wollenhaupt J, Huyen NTT, Weise C, Kalinowski J, Wahl MC, Fulde M, and Antelmann H. Redox-sensing under hypochlorite stress and infection conditions by the Rrf2-family repressor HypR in Staphylococcus aureus. Antioxid Redox Signal 29: 615636, 2018.

45. Loi VV, Harms M, Müller M, Huyen NTT, Hamilton CJ, Hochgräfe F, Pane-Farre J, and Antelmann H. Real-time imaging of the bacillithiol redox potential in the human pathogen Staphylococcus aureus using a genetically encoded bacilliredoxin-fused redox biosensor. Antioxid Redox Signal 26: 835-848, 2017.

46. Love MI, Huber W, and Anders S. Moderated estimation of fold change and dispersion for RNA-seq data with DESeq2. Genome Biol 15: 550, 2014.

47. Lowy FD. Staphylococcus aureus infections. N Engl J Med 339: 520-532, 1998.

48. Luebke JL, Shen J, Bruce KE, Kehl-Fie TE, Peng H, Skaar EP, and Giedroc DP. The CsoR-like sulfurtransferase repressor (CstR) is a persulfide sensor in Staphylococcus aureus. Mol Microbiol 94: 1343-1360, 2014.

49. Mäder U, Nicolas P, Depke M, Pane-Farre J, Debarbouille M, van der Kooi-Pol MM, Guerin C, Derozier S, Hiron A, Jarmer H, Leduc A, Michalik S, Reilman E, Schaffer M, Schmidt F, Bessieres P, Noirot P, Hecker M, Msadek T, Völker U, and van Dijl JM. Staphylococcus aureus transcriptome architecture: from laboratory to infectionmimicking conditions. PLoS Genet 12: e1005962, 2016.

50. Mayer S, Steffen W, Steuber J, and Gotz F. The Staphylococcus aureus NuoL-like protein MpsA contributes to the generation of membrane potential. J Bacteriol 197: 794806, 2015.

51. Miyauchi K, Adachi Y, Nagata Y, and Takagi M. Cloning and sequencing of a novel meta-cleavage dioxygenase gene whose product is involved in degradation of gammahexachlorocyclohexane in Sphingomonas paucimobilis. J Bacteriol 181: 6712-6719, 1999.

52. Monks TJ, Hanzlik RP, Cohen GM, Ross D, and Graham DG. Quinone chemistry and toxicity. Toxicol Appl Pharmacol 112: 2-16, 1992.

53. Moussaoui M, Miseviciene L, Anusevicius Z, Maroziene A, Lederer F, Baciou L, and Cenas N. Quinones and nitroaromatic compounds as subversive substrates of Staphylococcus aureus flavohemoglobin. Free Radic Biol Med 123: 107-115, 2018.

54. National Nosocomial Infections Surveillance System Report. National Nosocomial Infections Surveillance (NNIS) System Report, data summary from January 1992 through June 2004, issued October 2004. Am J Infect Control 32: 470-485, 2004.

55. Nguyen VD, Wolf C, Mäder U, Lalk M, Langer P, Lindequist U, Hecker M, and Antelmann H. Transcriptome and proteome analyses in response to 2-methylhydroquinone and 6-brom-2-vinyl-chroman-4-on reveal different degradation systems involved in the catabolism of aromatic compounds in Bacillus subtilis. Proteomics 7: 1391-1408, 2007.

56. Noto MJ, Burns WJ, Beavers WN, and Skaar EP. Mechanisms of pyocyanin toxicity and genetic determinants of resistance in Staphylococcus aureus. J Bacteriol 199: e00221-17, 2017.

57. O'Brien PJ. Molecular mechanisms of quinone cytotoxicity. Chem Biol Interact 80: 1-41, 1991.

58. O’Malley YQ, Reszka KJ, Spitz DR, Denning GM, and Britigan BE. Pseudomonas aeruginosa pyocyanin directly oxidizes glutathione and decreases its levels in airway 
epithelial cells. Am J Physiol Lung Cell Mol Physiol 287: L94-L103, 2004.

59. Ollis DL, Cheah E, Cygler M, Dijkstra B, Frolow F, Franken SM, Harel M, Remington SJ, Silman I, Schrag J, Sussman JL, Verschueren KHG, and Goldman A. The alpha/beta hydrolase fold. Protein Eng 5: 197-211, 1992.

60. Palm GJ, Khanh Chi B, Waack P, Gronau K, Becher D, Albrecht D, Hinrichs W, Read RJ, and Antelmann H. Structural insights into the redox-switch mechanism of the MarR/DUF24-type regulator HypR. Nucleic Acids Res 40: 4178-4192, 2012.

61. Panmanee W, Vattanaviboon P, Poole LB, and Mongkolsuk S. Novel organic hydroperoxide-sensing and responding mechanisms for OhrR, a major bacterial sensor and regulator of organic hydroperoxide stress. J Bacteriol 188: 1389-1395, 2006.

62. Perera IC and Grove A. Molecular mechanisms of ligandmediated attenuation of DNA binding by MarR family transcriptional regulators. J Mol Cell Biol 2: 243-254, 2010.

63. Pfeifer-Sancar K, Mentz A, Ruckert C, and Kalinowski J. Comprehensive analysis of the Corynebacterium glutamicum transcriptome using an improved RNAseq technique. BMC Genomics 14: 888, 2013.

64. Phillips-Jones MK and Harding SE. Antimicrobial resistance (AMR) nanomachines-mechanisms for fluoroquinolone and glycopeptide recognition, efflux and/or deactivation. Biophys Rev 10: 347-362, 2018.

65. Pi $\mathrm{H}$ and Helmann JD. Genome-wide characterization of the Fur regulatory network reveals a link between catechol degradation and bacillibactin metabolism in Bacillus subtilis. mBio 9: pii:e01451-18, 2018.

66. Rosenblum ED and Tyrone S. Serology, density, and morphology of staphylococcal phages. J Bacteriol 88: 17371742, 1964.

67. Soutourina O, Dubrac S, Poupel O, Msadek T, and MartinVerstraete I. The pleiotropic CymR regulator of Staphylococcus aureus plays an important role in virulence and stress response. PLoS Pathog 6: e1000894, 2010.

68. Tam le T, Eymann C, Albrecht D, Sietmann R, Schauer F, Hecker M, and Antelmann H. Differential gene expression in response to phenol and catechol reveals different metabolic activities for the degradation of aromatic compounds in Bacillus subtilis. Environ Microbiol 8: 1408-1427, 2006.

69. Töwe S, Leelakriangsak M, Kobayashi K, Van Duy N, Hecker M, Zuber P, and Antelmann H. The MarR-type repressor MhqR (YkvE) regulates multiple dioxygenases/ glyoxalases and an azoreductase which confer resistance to 2-methylhydroquinone and catechol in Bacillus subtilis. Mol Microbiol 66: 40-54, 2007.

70. Truong-Bolduc QC, Dunman PM, Strahilevitz J, Projan SJ, and Hooper DC. MgrA is a multiple regulator of two new efflux pumps in Staphylococcus aureus. J Bacteriol 187: 2395-2405, 2005.

71. Unden $\mathrm{G}$ and Bongaerts J. Alternative respiratory pathways of Escherichia coli: energetics and transcriptional regulation in response to electron acceptors. Biochim Biophys Acta 1320: 217-234, 1997.

72. Van Loi V, Busche T, Preuss T, Kalinowski J, Bernhardt J, and Antelmann H. The AGXX antimicrobial coating causes a thiol-specific oxidative stress response and protein $S$ bacillithiolation in Staphylococcus aureus. Front Microbiol 9: 3037, 2018.

73. Wetzstein M, Völker U, Dedio J, Löbau S, Zuber U, Schiesswohl M, Herget C, Hecker M, and Schumann W. Cloning, sequencing, and molecular analysis of the dnaK locus from Bacillus subtilis. J Bacteriol 174: 3300-3310, 1992.

74. Wichelhaus TA, Boddinghaus B, Besier S, Schafer V, Brade V, and Ludwig A. Biological cost of rifampin resistance from the perspective of Staphylococcus aureus. Antimicrob Agents Chemother 46: 3381-3385, 2002.

75. Wilkinson SP and Grove A. Ligand-responsive transcriptional regulation by members of the MarR family of winged helix proteins. Curr Issues Mol Biol 8: 51-62, 2006.

76. Winter J, Ilbert M, Graf PC, Ozcelik D, and Jakob U. Bleach activates a redox-regulated chaperone by oxidative protein unfolding. Cell 135: 691-701, 2008.

77. Wosilait WD and Nason A. Pyridine nucleotide-menadione reductase from Escherichia coli. J Biol Chem 208: 785798, 1954.

78. Zeden MS, Schuster CF, Bowman L, Zhong Q, Williams HD, and Grundling A. Cyclic di-adenosine monophosphate (c-di-AMP) is required for osmotic regulation in Staphylococcus aureus but dispensable for viability in anaerobic conditions. J Biol Chem 293: 3180-3200, 2018.

Address correspondence to: Prof. Haike Antelmann Institute for Biology-Microbiology Freie Universität Berlin Königin-Luise-Strasse 12-16 Berlin D-14195 Germany

E-mail: haike.antelmann@fu-berlin.de

Date of first submission to ARS Central, February 5, 2019; date of final revised submission, June 18, 2019; date of acceptance, July 5, 2019.

$$
\begin{aligned}
& \text { Abbreviations Used } \\
\mathrm{BSH} & =\text { bacillithiol } \\
\mathrm{CFU} & =\text { colony-forming unit } \\
\mathrm{DTT} & =\text { dithiothreitol } \\
\mathrm{EMSA} & =\text { electrophoretic mobility shift assay } \\
\mathrm{H}_{2} \mathrm{O}_{2} & =\text { hydrogen peroxide } \\
\mathrm{HOCl} & =\text { hypochlorous acid } \\
\mathrm{LB} & =\text { Luria-Bertani } \\
\mathrm{MHQ} & =\text { methylhydroquinone } \\
\mathrm{NaOCl} & =\text { sodium hypochlorite } \\
\mathrm{ROS} & =\text { reactive oxygen species } \\
\mathrm{TSS} & =\text { transcription start site } \\
\mathrm{wHTH} & =\text { winged helix-turn-helix }
\end{aligned}
$$

